\title{
Odd-frequency pairing in normal-metal/superconductor junctions
}

\author{
Y. Tanaka, ${ }^{1,2}$ Y. Tanuma, ${ }^{3}$ and A. A. Golubov ${ }^{4}$ \\ ${ }^{1}$ Department of Applied Physics, Nagoya University, Nagoya 464-8603, Japan \\ ${ }^{2}$ CREST Japan Science and Technology Cooperation (JST), Nagoya 464-8603, Japan \\ ${ }^{3}$ Institute of Physics, Kanagawa University, 3-7-1, Rokkakubashi, Kanagawa-ku, Yokohama 221-8686, Japan \\ ${ }^{4}$ Faculty of Science and Technology, University of Twente, Enschede, 7500AE, The Netherlands
}

(Received 16 May 2007; published 29 August 2007)

We theoretically study the induced odd-frequency pairing states in ballistic normal-metal/superconductor (N/S) junctions where a superconductor has even-frequency symmetry in the bulk and a normal-metal layer has an arbitrary length. Using the quasiclassical Green's function formalism, we demonstrate that, quite generally, the pair amplitude in the junction has an admixture of an odd-frequency component due to the breakdown of translational invariance near the N/S interface where the pair potential acquires spatial dependence. If a superconductor has an even-parity pair potential (spin-singlet $s$-wave or spin-singlet $d_{x y}$-wave state), the odd-frequency pairing component with odd parity is induced near the N/S interface, while in the case of an odd-parity pair potential (spin-triplet $p_{x}$ wave) the odd-frequency component with even parity is generated. We show that in conventional $s$-wave junctions, the amplitude of the odd-frequency pairing state is strongest in the case of a full-transparency N/S interface and is enhanced at energies corresponding to the peaks in the local density of states (LDOS). In $p_{x^{-}}$and $d_{x y}$-wave junctions, the amplitude of the odd-frequency component on the $\mathrm{S}$ side of the N/S interface is enhanced at zero energy where the midgap Andreev resonant state (MARS) appears due to the sign change of the pair potential. The odd-frequency component extends into the $\mathrm{N}$ region and exceeds the even-frequency component at energies corresponding to the LDOS peak positions, including the MARS. At the edge of the $\mathrm{N}$ region the odd-frequency component is nonzero while the even-frequency one vanishes. We show that the concept of the odd-frequency pairing state plays a pivotal role to interpret a number of phenomena in nonuniform superconducting systems, like McMillan-Rowell and midgap Andreev resonance states.

DOI: 10.1103/PhysRevB.76.054522

\section{INTRODUCTION}

The odd-frequency superconducting pairing state, characterized by a pair amplitude that is an odd function of energy or Matsubara frequency, was first predicted by Berezinskii ${ }^{1}$ and has attracted a lot of interest recently. Although the existence of odd-frequency pairing in bulk uniform systems is not fully established yet, ${ }^{2-6}$ there is a number of proposals to realize it in superconducting junctions. The realization of the odd-frequency pairing state without a finite pair potential was proposed by Bergeret, Volkov, and Efetov in Ref. 7 in ferromagnet/superconductor heterostructures with inhomogeneous magnetization and several related works have been presented up to now. ${ }^{8-10}$ In particular, it was predicted that the local density of states (LDOS) in the ferromagnet is enhanced in the presence of the odd-frequency pairing. ${ }^{11}$

Recently, it was shown that an odd-frequency pairing state is possible even without magnetic ordering. Tanaka and Golubov predicted that the odd-frequency pair amplitude can be induced in a diffusive normal metal attached to a spin-triplet superconductor. ${ }^{12}$ According to this study, the origin of the anomalous proximity effect specific to spin-triplet $p$-wave superconductor junctions ${ }^{13}$ is the realization of the oddfrequency pairing state in a diffusive normal metal. It is also clarified that the penetration of the midgap Andreev resonant state $^{14,15}$ (MARS) into the diffusive normal metal is a manifestation of the existence of the odd-frequency spin-triplet $s$-wave superconducting state. The MARS is the well-known resonant state specific to unconventional superconductors with sign change of the pair potential on the Fermi surface, and was observed experimentally in various materials. ${ }^{16}$
PACS number(s): 74.45.+c, 74.50.+r, 74.20.Rp

Furthermore, it was predicted very recently ${ }^{17,18}$ that, due to spatial variation of the pair potential near a normal-metal/ superconductor $(\mathrm{N} / \mathrm{S})$ interface, ${ }^{19}$ the odd-frequency pairing state can be induced even in a conventional ballistic N/S system without spin-triplet ordering. By studying infinite normal-metal/infinite superconductor (N/S) junctions, it was shown that, quite generally, the spin-singlet even-parity (spin-triplet odd-parity) pair potential in the superconductor induces an odd-frequency pairing component with spinsinglet odd-parity (spin-triplet even-parity) near the N/S interface. ${ }^{17}$ The magnitude of the induced odd-frequency component is enhanced in the presence of the MARS due to the sign change of the anisotropic pair potential at the interface. In Ref. 17, only the generation of the odd-frequency component at the $\mathrm{S}$ side of the N/S interface was studied by Tanaka et al. Therefore the questions remain how this component extends into the $\mathrm{N}$ region and how it manifests itself in the properties of the normal metal. In a semi-infinite ballistic normal metal attached to a superconductor, the LDOS normalized by its value in the normal state is always unity. This well-known property of the LDOS is due to the absence of interference between electrons and Andreev reflected holes in a semi-infinite $\mathrm{N}$ metal. In this case, the LDOS cannot be used to characterize the superconducting correlations in a normal metal. Thus, in order to understand manifestations of the induced odd-frequency pairing state in the $\mathrm{N}$ metal in a much clearer way, it is necessary to study junctions with finite length of the $\mathrm{N}$ region.

In the present paper, using quasiclassical Green's function theory, we study the pair amplitude and the LDOS at the N/S interface when the $\mathrm{N}$ region has finite thickness $L$. The spa- 
tial dependence of the pair potential is determined selfconsistently. For convenience of the actual numerical calculation, we have used the boundary condition in the Ricatti parametrization. ${ }^{20}$ The superconductor is assumed to have the conventional even-frequency pairing state in the bulk, being in the spin-singlet even- ( $s$-wave or $d_{x y}$-wave symmetry) or the spin-triplet odd-parity state ( $p_{x}$-wave symmetry). We show that, quite generally, the spatial variation of the pair potential and the proximity effect lead to the generation of the odd-frequency component near the N/S interface and on the $\mathrm{N}$ side. Moreover, when the superconductor is in the even-parity (odd-parity) state, the resulting odd-frequency component is odd parity (even parity) in order to conserve the spin component. In the absence of the MARS, as in the case of spin-singlet $s$-wave junctions, the magnitude of the odd-frequency component of the pair amplitude is suppressed when the transmission coefficient through the interface decreases. The resulting odd-frequency pair amplitude has its maximum value at the interface. At the edge of the $\mathrm{N}$ region, the odd-frequency component is always absent, as well as in the $\mathrm{S}$ region far away from the interface. The LDOS is suppressed around $\varepsilon=0$, where $\varepsilon$ is the quasiparticle energy measured from the Fermi level. For large magnitude of $L$, the resulting LDOS has an oscillatory $\varepsilon$ dependence. The amplitude of the odd-frequency pair amplitude can exceed that of the even-frequency one at some $\varepsilon$ values. For the case of spin-triplet $p_{x}$-wave and spin-singlet $d_{x y}$-wave junctions, the amplitude of the odd-frequency component at the $\mathrm{S}$ side of the N/S interface is much larger than that of the even-frequency pair amplitude. This is due to the fact that the presence of the MARS at the interface ${ }^{14}$ enhances the amplitude of the odd-frequency pairing state as shown in Ref. 17. At the edge of the $\mathrm{N}$ region, the even-frequency component is always absent and only the odd-frequency component is nonzero. At $\varepsilon=0$ the resulting odd-frequency component always exceeds the even-frequency one.

The organization of the present paper is as follows. In Sec. II, we introduce the model and the quasiclassical Green's function formalism. In Sec. III, the results of the numerical calculations are discussed for the case of spinsinglet $s$-wave, spin-triplet $p_{x}$-wave, and spin-singlet $d_{x y}$-wave junctions. In Sec. IV, the conclusions and outlook are presented.

\section{MODEL AND FORMULATION}

In the following, we consider a N/S junction as the simplest example of a nonuniform superconducting system without impurity scattering. Both cases of spin-triplet odd-parity and spin-singlet even-parity symmetries are considered in the superconductor. In the spin-triplet superconductor, we choose $S_{z}=0$ for simplicity. We assume a thin insulating barrier located at the N/S interface $(x=0)$ with $\mathrm{N}(-L<x<0)$ and $\mathrm{S}(x>0)$ modeled by a $\delta$-function $H \delta(x)$, where $H$ is the strength of the $\delta$-function potential. The length of the normal region is $L$. The reflection coefficient of the junction for the quasiparticle for injection angle $\theta$ is given by $R=Z^{2} /\left(Z^{2}\right.$ $\left.+4 \cos ^{2} \theta\right)$ with $Z=2 H / v_{F}$, where $\theta(-\pi / 2<\theta<\pi / 2)$ is measured from the normal to the interface and $v_{F}$ is the Fermi velocity.
The quasiclassical Green's functions ${ }^{21}$ in a normal metal $(\mathrm{N})$ and a superconductor (S) in the Matsubara frequency representation are parametrized as

$$
\hat{g}_{ \pm}^{(i)}=f_{1 \pm}^{(i)} \hat{\tau}_{1}+f_{2 \pm}^{(i)} \hat{\tau}_{2}+g_{ \pm}^{(i)} \hat{\tau}_{3}, \quad\left(\hat{g}_{ \pm}^{(i)}\right)^{2}=\hat{1}
$$

where the subscripts $i(=N, S)$ refer to $\mathrm{N}$ and $\mathrm{S}$, respectively. Here, $\hat{\tau}_{j}(j=1,2,3)$ are Pauli matrices and $\hat{1}$ is a unit matrix. The subscript $+(-)$ denotes the left (right) going quasiparticles. ${ }^{22}$ The functions $\hat{g}_{ \pm}^{(i)}$ satisfy the Eilenberger equation $^{23}$

$$
i v_{F x} \hat{g}_{ \pm}^{(i)}=\mp\left[\hat{H}_{ \pm}, \hat{g}_{ \pm}^{(i)}\right]
$$

with

$$
\hat{H}_{ \pm}=i \omega_{n} \hat{\tau}_{3}+i \bar{\Delta}_{ \pm}(x) \hat{\tau}_{2} .
$$

Here $v_{F x}$ is the $x$ component of the Fermi velocity, $\omega_{n}$ $=2 \pi T(n+1 / 2)$ is the Matsubara frequency, $n$ is an integer number, and $T$ is the temperature. $\bar{\Delta}_{+}(x)\left[\bar{\Delta}_{-}(x)\right]$ is the effective pair potential for left (right) going quasiparticles. In the $\mathrm{N}$ region, $\bar{\Delta}_{ \pm}(x)$ is set to zero due to the absence of a pairing interaction in the $\mathrm{N}$ metal. The above Green's functions can be expressed as

$$
\begin{aligned}
f_{1 \pm}^{(i)} & = \pm i\left[F_{ \pm}^{(i)}(x)+D_{ \pm}^{(i)}(x)\right] /\left[1-D_{ \pm}^{(i)}(x) F_{ \pm}^{(i)}(x)\right], \\
f_{2 \pm}^{(i)} & =-\left[F_{ \pm}^{(i)}(x)-D_{ \pm}^{(i)}(x)\right] /\left[1-D_{ \pm}^{(i)}(x) F_{ \pm}^{(i)}(x)\right], \\
g_{ \pm}^{(i)} & =\left[1+D_{ \pm}^{(i)}(x) F_{ \pm}^{(i)}(x)\right] /\left[1-D_{ \pm}^{(i)}(x) F_{ \pm}^{(i)}(x)\right],
\end{aligned}
$$

where $D_{ \pm}^{(i)}(x)$ and $F_{ \pm}^{(i)}(x)$ satisfy the Ricatti equations ${ }^{20}$ in the $\mathrm{N}$ region,

$$
\begin{gathered}
v_{F x} \partial_{x} D_{ \pm}^{(N)}(x)=-2 \omega_{n} D_{ \pm}^{(N)}(x), \\
v_{F x} \partial_{x} F_{ \pm}^{(N)}(x)=2 \omega_{n} F_{ \pm}^{(N)}(x),
\end{gathered}
$$

and in the $\mathrm{S}$ region,

$$
\begin{aligned}
& v_{F x} \partial_{x} D_{ \pm}^{(S)}(x)=-\bar{\Delta}_{ \pm}(x)\left[1-\left(D_{ \pm}^{(S)}(x)\right)^{2}\right]+2 \omega_{n} D_{ \pm}^{(S)}(x), \\
& v_{F x} \partial_{x} F_{ \pm}^{(S)}(x)=-\bar{\Delta}_{ \pm}(x)\left[1-\left(F_{ \pm}^{(S)}(x)\right)^{2}\right]-2 \omega_{n} F_{ \pm}^{(S)}(x),
\end{aligned}
$$

respectively.

The boundary conditions at the edge of $\mathrm{N}$ region, $x=-L$, have the form

$$
F_{+}^{(N)}(-L)=-D_{-}^{(N)}(-L), \quad F_{-}^{(N)}(-L)=-D_{+}^{(N)}(-L) .
$$

The boundary conditions at the N/S interface, $x=0$, are

$$
F_{ \pm}^{(S)}(0)=-\frac{(1-R) D_{ \pm}^{(N)}(0)+\left[R+D_{+}^{(N)}(0) D_{-}^{(N)}(0)\right] D_{+}^{(S)}(0)}{\left[1+R D_{+}^{(N)}(0) D_{-}^{(N)}(0)\right]+(1-R) D_{\mp}^{(N)}(0) D_{\mp}^{(S)}(0)}
$$

and 


$$
F_{ \pm}^{(N)}(0)=-\frac{(1-R) D_{ \pm}^{(S)}(0)+\left[R+D_{+}^{(S)}(0) D_{-}^{(S)}(0)\right] D_{+}^{(N)}(0)}{\left[1+R D_{+}^{(S)}(0) D_{-}^{(S)}(0)\right]+(1-R) D_{\mp}^{(N)}(0) D_{+}^{(S)}(0)}
$$

where $R$ is the reflection coefficient at the interface. Since there is no pair potential in the $\mathrm{N}$ region, the solutions for the spatial dependence of above functions can be easily found:

$$
\begin{gathered}
D_{-}^{(N)}(x)=-A t^{-1}, \quad D_{+}^{(N)}(x)=-B t^{-1}, \\
F_{+}^{(N)}(x)=A t, \quad F_{-}^{(N)}(x)=B t,
\end{gathered}
$$

with $t=\exp [(x+L) / \xi]$ and $\xi=\hbar v_{F x} / 2\left|\omega_{n}\right|$. The constants $A$ and $B$ are given by

$$
\begin{aligned}
& A=\frac{-2(1-R) D_{+}^{(S)}(0) t_{0}}{\Lambda+\sqrt{\Lambda^{2}+4(1-R)^{2} t_{0}^{2} D_{+}^{(S)}(0) D_{-}^{(S)}(0)}}, \\
& B=\frac{-2(1-R) D_{-}^{(S)}(0) t_{0}}{\Lambda+\sqrt{\Lambda^{2}+4(1-R)^{2} t_{0}^{2} D_{+}^{(S)}(0) D_{-}^{(S)}(0)}},
\end{aligned}
$$

with

$$
\Lambda=t_{0}^{2}\left[1+R D_{+}^{(S)}(0) D_{-}^{(S)}(0)\right]-\left[R+D_{+}^{(S)}(0) D_{-}^{(S)}(0)\right]
$$

and $t_{0}=\exp (L / \xi)$. After simple manipulation, we obtain $f_{1+}^{(N)}$, $f_{2 \pm}^{(N)}$, and $g_{ \pm}^{(N)}$,

$$
\begin{array}{ll}
f_{1+}^{(N)}=-i(A t-B / t) /(1+A B), & f_{1-}^{(N)}=i(B t-A / t) /(1+A B), \\
f_{2+}^{(N)}=-(A t+B / t) /(1+A B), & f_{2-}^{(N)}=-(B t+A / t) /(1+A B),
\end{array}
$$

$$
g_{+}^{(N)}=g_{-}^{(N)}=(1-A B) /(1+A B) .
$$

Note that, as follows from Eq. (14), the functions $g_{+}^{(N)}$ and $g_{-}^{(N)}$ are spatially independent. For $Z=0$, since $A$ $=-D_{+}^{(S)}(0) / t_{0}$ and $B=-D_{-}^{(S)}(0) / t_{0}$ are satisfied, then Eqs. (13) and (14) can be expressed much more simply as

$$
\begin{gathered}
f_{1 \pm}^{(N)}= \pm i \frac{D_{ \pm}^{(S)}(0) t-D_{ \pm}^{(S)}(0) / t}{t_{0}+D_{ \pm}^{(S)}(0) D_{ \pm}^{(S)}(0) / t_{0}}, \\
f_{2 \pm}^{(N)}=\frac{D_{ \pm}^{(S)}(0) t+D_{ \pm}^{(S)}(0) / t}{t_{0}+D_{ \pm}^{(S)}(0) D_{ \pm}^{(S)}(0) / t_{0}},
\end{gathered}
$$

and

$$
g_{+}^{(N)}=g_{-}^{(N)}=\frac{t_{0}-D_{ \pm}^{(S)}(0) D_{ \pm}^{(S)}(0) / t_{0}}{t_{0}+D_{ \pm}^{(S)}(0) D_{ \pm}^{(S)}(0) / t_{0}} .
$$

Here, we consider the situation without mixing of different symmetry channels for the pair potential. Then the pair potential $\bar{\Delta}_{ \pm}(x)$ is expressed by

$$
\bar{\Delta}_{ \pm}(x)=\Delta(x) \Phi_{ \pm}(\theta) \Theta(x)
$$

with form factor $\Phi_{ \pm}(\theta)$ given by $\Phi_{ \pm}(\theta)=1, \pm \sin 2 \theta$, and $\pm \cos \theta$ for $s$-wave, $d_{x y}$-wave, and $p_{x}$-wave superconductors, respectively. For the $s$-wave case, $D_{+}^{(i)}(x)=D_{-}^{(i)}(x)$ are satis- fied, while for the $p_{x}$-wave and $d_{x y}$-wave cases, $D_{+}^{(i)}(x)$ $=-D_{-}^{(i)}(x)$ are satisfied.

$\Delta(x)$ is determined self-consistently by the following equation:

$$
\Delta(x)=\frac{2 T}{\ln \left(T / T_{C}\right)+\sum_{n \geqslant 0} \frac{1}{n+1 / 2}} \sum_{n \geqslant 0} \int_{-\pi / 2}^{\pi / 2} d \theta G(\theta) f_{2+}
$$

with $G(\theta)=1$ for the $s$-wave case and $2 \Phi(\theta)$ for other cases. ${ }^{24} T_{C}$ is the transition temperature of the superconductor. We introduce a cutoff of the summation of $n, n_{\max }$, in Eq. (19), where $n_{\max }$ is the maximum integer that satisfies

$$
n_{\max } \leqslant \frac{\omega_{D}}{2 \pi T} .
$$

The condition in the bulk is $\Delta(\infty)=\Delta_{0}$. Since the pair potential $\bar{\Delta}(x)$ is a real number, the resulting $f_{1 \pm}$ is an imaginary number and $f_{2 \pm}$ is a real one.

Before performing actual numerical calculations, we now discuss general properties of the pair amplitude. In the following, we explicitly write $f_{1 \pm}^{(i)}=f_{1 \pm}^{(i)}\left(\omega_{n}, \theta\right), f_{2 \pm}^{(i)}=f_{2 \pm}^{(i)}\left(\omega_{n}, \theta\right)$, $F_{ \pm}^{(i)}=F_{ \pm}^{(i)}\left(x, \omega_{n}, \theta\right)$, and $D_{ \pm}^{(i)}=D_{ \pm}^{(i)}\left(x, \omega_{n}, \theta\right)$. For the limit $x$ $=\infty$, we obtain

$$
f_{1 \pm}^{(S)}\left(\omega_{n}, \theta\right)=0, \quad f_{2 \pm}^{(S)}\left(\omega_{n}, \theta\right)=\frac{\Delta_{0} \Phi_{ \pm}(\theta)}{\sqrt{\omega_{n}^{2}+\Delta_{0}^{2} \Phi_{ \pm}^{2}\left(\theta_{ \pm}\right.}} .
$$

Note that $f_{1+}^{(i)}\left(\omega_{n}, \theta\right)$ becomes finite due to the spatial variation of the pair potential and it does not exist in the bulk. From Eqs. (4) and (5), we can show that $D_{ \pm}^{(i)}\left(x,-\omega_{n}, \theta\right)$ $=1 / D_{ \pm}^{(i)}\left(x, \omega_{n}, \theta\right)$ and $F_{ \pm}^{(i)}\left(x,-\omega_{n}, \theta\right)=1 / F_{ \pm}^{(i)}\left(x, \omega_{n}, \theta\right)$. After simple manipulation, we obtain

$$
f_{1 \pm}^{(i)}\left(\omega_{n}, \theta\right)=-f_{1 \pm}^{(i)}\left(-\omega_{n}, \theta\right), \quad f_{2 \pm}^{(i)}\left(\omega_{n}, \theta\right)=f_{2 \pm}^{(i)}\left(-\omega_{n}, \theta\right),
$$

for any $x$. It is remarkable that the functions $f_{1 \pm}^{(i)}\left(\omega_{n}, \theta\right)$ and $f_{2 \pm}^{(i)}\left(\omega_{n}, \theta\right)$ correspond to odd-frequency and even-frequency components of the pair amplitude, respectively. ${ }^{8,17}$ The function $f_{1 \pm}^{(1)}\left(\omega_{n}, \theta\right)$ describes the odd-frequency component of the pair amplitude penetrating from the superconductor.

Next, we discuss the parity of these pair amplitudes. The even-parity (odd-parity) pair amplitude should satisfy the relation $f_{j \pm}^{(i)}\left(\omega_{n}, \theta\right)=f_{j \mp}^{(i)}\left(\omega_{n},-\theta\right) \quad\left[f_{j \pm}^{(i)}\left(\omega_{n}, \theta\right)=-f_{j \mp}^{(i)}\left(\omega_{n},-\theta\right)\right]$, with $j=1,2$. For an even-parity (odd-parity) superconductor, $\Phi_{ \pm}(-\theta)=\Phi_{\mp}(\theta)\left[\Phi_{ \pm}(-\theta)=-\Phi_{\mp}(\theta)\right]$. Then, we can show that for the even-parity case

$$
D_{ \pm}^{(i)}\left(x, \omega_{n},-\theta\right)=D_{\mp}^{(i)}\left(x, \omega_{n}, \theta\right), \quad F_{ \pm}^{(i)}\left(x, \omega_{n},-\theta\right)=F_{+}^{(i)}\left(x, \omega_{n}, \theta\right)
$$

and for the odd-parity case

$$
D_{ \pm}^{(i)}\left(x, \omega_{n},-\theta\right)=-D_{\mp}^{(i)}\left(x, \omega_{n}, \theta\right),
$$




$$
F_{ \pm}^{(i)}\left(x, \omega_{n},-\theta\right)=-F_{\mp}^{(i)}\left(x, \omega_{n}, \theta\right),
$$

respectively.

The resulting $f_{1 \pm}^{(i)}\left(\omega_{n}, \theta\right)$ and $f_{2 \pm}^{(i)}\left(\omega_{n}, \theta\right)$ satisfy

$$
\begin{gathered}
f_{1 \pm}^{(i)}\left(\omega_{n}, \theta\right)=-f_{1 \mp}^{(i)}\left(\omega_{n},-\theta\right), \\
f_{2 \pm}^{(i)}\left(\omega_{n}, \theta\right)=f_{2 \mp}^{(i)}\left(\omega_{n},-\theta\right)
\end{gathered}
$$

for an even-parity superconductor and

$$
\begin{aligned}
& f_{1 \pm}^{(i)}\left(\omega_{n}, \theta\right)=f_{1 \mp}^{(i)}\left(\omega_{n},-\theta\right), \\
& f_{2 \pm}^{(i)}\left(\omega_{n}, \theta\right)=-f_{2 \mp}^{(i)}\left(\omega_{n},-\theta\right)
\end{aligned}
$$

for an odd-parity superconductor. ${ }^{17}$ Note that the parity of the odd-frequency component $f_{1 \pm}^{(i)}\left(\omega_{n}, \theta\right)$ is always different from that in the bulk superconductor. It should be also noted that, as seen from Eq. (13), the odd-frequency component $f_{1 \pm}^{(i)}\left(\omega_{n}, \theta\right)$ is purely an imaginary quantity.

The underlying physics behind this formal property is the following. Due to the breakdown of translational invariance near the N/S interface, the pair potential $\bar{\Delta}(x)$ acquires a spatial dependence which leads to the coupling between even-parity and odd-parity states. Since the bulk pair potential has an even-frequency symmetry, the Fermi-Dirac statistics requires that the order parameter component induced near the interface should be odd in frequency. The phase of the induced pair amplitude undergoes a $\pi / 2$ shift from that in the bulk $\mathrm{S}$, thus removing the internal phase shift between the even- and odd-frequency components and making the interface-induced state compatible with time-reversal invariance. As a result, the function $f_{1 \pm}^{(i)}\left(\omega_{n}, \theta\right)$ becomes a purely imaginary number. ${ }^{17}$

Let us now focus on the values of the pair amplitudes at the edge of the $\mathrm{N}$ region (at $x=-L$ ). We concentrate on two extreme cases with (I) $\Phi_{+}(\theta)=\Phi_{-}(\theta)$ and (II) $\Phi_{+}(\theta)=$ $-\Phi_{-}(\theta)$. In case I, the MARS is absent since there is no sign change of the pair potential experienced by the quasiparticle at the interface. Then the relation $D_{+}^{(N)}(x)=D_{-}^{(N)}(x)$ holds. On the other hand, in case II, the MARS is generated near the interface due to the sign change of the pair potential and the relation $D_{+}^{(N)}(x)=-D_{-}^{(N)}(x)$ is satisfied..$^{14}$ At the edge $x=-L$, it is easy to show that $\bar{F}_{ \pm}^{(N)}(-L)=-D_{ \pm}^{(N)}(-L)$ for the former case and $F_{ \pm}^{(N)}(-L)=D_{ \pm}^{(N)}(-L)$ for the latter. As a result, $f_{1 \pm}^{(N)}(-L)$ $=0$ for case I and $f_{2 \pm}^{(N)}(-L)=0$ for case II. Thus we can conclude that in the absence of the MARS only the evenfrequency pairing component exists at $x=-L$, and in the presence of the MARS only the odd-frequency one.

The generation of the odd-frequency component $f_{1 \pm}^{(N(S))}$ is a very general feature. In the extreme limit with $R=1, f_{1 \pm}^{(N)}$ becomes zero due to the absence of the proximity effect. In this case, $f_{1 \pm}^{(S)}$ at the $\mathrm{S}$ side of the interface becomes

$$
f_{1 \pm}^{(S)}= \pm i \frac{-D_{ \pm}^{(S)}(0)+D_{+}^{(S)}(0)}{1+D_{+}^{(S)}(0) D_{-}^{(S)}(0)} .
$$

Only when $D_{+}^{(S)}(0)=D_{-}^{(S)}$ is satisfied does $f_{1 \pm}^{(S)}$ vanish. It should also be noted that the generation of $f_{1 \pm}^{(N(S))}$ is robust against the detailed features of the spatial dependence of the pair potential. Although we have determined the spatial dependence of the pair potential self-consistently, the generation of $f_{1 \pm}^{(N(S))}$ can be shown even if we ignore the spatial dependence of $\bar{\Delta}_{ \pm}(x)$. In such a case, $D_{ \pm}^{(S)}(0)$ is given as

$$
D_{ \pm}^{(S)}(0)=\frac{\Delta_{0} \Phi_{ \pm}\left(\theta_{ \pm}\right)}{\omega_{n}+\sqrt{\omega_{n}^{2}+\Delta_{0}^{2} \Phi_{ \pm}^{2}\left(\theta_{ \pm}\right)}} .
$$

In order to understand the angular dependence of the pair amplitude in more detail, we define $\hat{f}_{1}^{(i)}$ and $\hat{f}_{2}^{(i)}$ for $-\pi / 2$ $<\theta<3 \pi / 2$ with $\hat{f}_{1(2)}^{(i)}=f_{1(2)+}^{(i)}(\theta)$ for $-\pi / 2<\theta<\pi / 2$ and $\hat{f}_{1(2)}^{(i)}=f_{1(2)-}^{(i)}(\pi-\theta)$ for $\pi / 2<\theta<3 \pi / 2$. We decompose $\hat{f}_{1(2)}^{(i)}$ into various angular momentum components as follows:

$$
\hat{f}_{1(2)}^{(i)}=\sum_{m} S_{m}^{(1(2))} \sin (m \theta)+\sum_{m} C_{m}^{(1(2))} \cos (m \theta),
$$

with $m=2 l+1$ for the odd-parity case and $m=2 l$ for the evenparity case with integer $l \geqslant 0$, where $l$ is the quantum number of the angular momentum. Here, $C_{m}^{(1(2))}$ and $S_{m}^{(1(2))}$ are defined for all $x$. It is straightforward to show that the only nonzero components are (1) $C_{2 l}^{(2)}$ and $C_{2 l+1}^{(1)}$ for even-parity superconductor without sign change at the interface, i.e., $s$-wave or

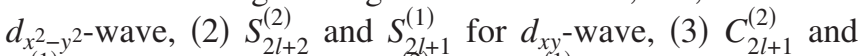
$C_{2 l}^{(1)}$ for $p_{x}$-wave, and (4) $S_{2 l+1}^{(2)}$ and $S_{2 l+2}^{(1)}$ for $p_{y}$-wave junctions, respectively. The allowed angular momenta for oddfrequency components are $2 l+1,2 l+1,2 l$, and $2 l+2$ corresponding to each of the above four cases.

In order to get better insight into the spectral property of the odd-frequency pair amplitude, we perform an analytical continuation from the Matsubara frequency $\omega_{n}$ to the quasiparticle energy $\varepsilon$ measured from the chemical potential. The retarded Green's function corresponding to Eq. (1) is defined as $\hat{g}_{ \pm}^{(i) R}=f_{1 \pm}^{(i) R} \hat{\tau}_{1}+f_{2 \pm}^{(i) R} \hat{\tau}_{2}+g_{ \pm}^{(i) R} \hat{\tau}_{3}$. One can show that $f_{1 \pm}^{(i) R}(-\varepsilon)$ $=-\left[f_{1 \pm}^{(i) R}(\varepsilon)\right]^{*}, f_{2 \pm}^{(i) R}(-\varepsilon)=\left[f_{2 \pm}^{(i) R}(\varepsilon)\right]^{*}$, and $g_{ \pm}^{(i) R}(-\varepsilon)=\left[g_{ \pm}^{(i) R}(\varepsilon)\right]^{*}$. The LDOS $\rho(\varepsilon)$ at the N/S interface at $x=0$ normalized to its value in the normal state is given by

$$
\rho(\varepsilon)=\int_{-\pi / 2}^{\pi / 2} d \theta \operatorname{Re}\left(\frac{g_{+}^{(i) R}(\varepsilon)+g_{-}^{(i) R}(\varepsilon)}{2 \pi}\right) .
$$

In the following, we self-consistently calculate the spatial dependence of the pair potential and the pair amplitude in the Matsubara representation. After that we calculate the spectral properties of pair amplitudes and the LDOS. For actual calculations, we choose spin-singlet $s$-wave, spin-triplet $p_{x}$-wave, and spin-singlet $d_{x y}$-wave states in a superconductor and fix the temperature $T=0.05 T_{C}$. The cutoff frequency $\omega_{D}$ is set to be $\omega_{D} / 2 \pi T_{C}=1$. The length of the normal region $L$ is measured in units of $L_{0}=v_{F} / 2 \pi T_{C}$.

\section{RESULTS}

\section{A. $s$-wave pair potential}

First we focus on the $s$-wave superconductor junctions as shown in Fig. 1. By changing the length $L$ of the $\mathrm{N}$ region and the transparency at the interface, we calculate the spatial 
(a) $\mathrm{Z}=0, \mathrm{~L}=\mathrm{L}_{0}$
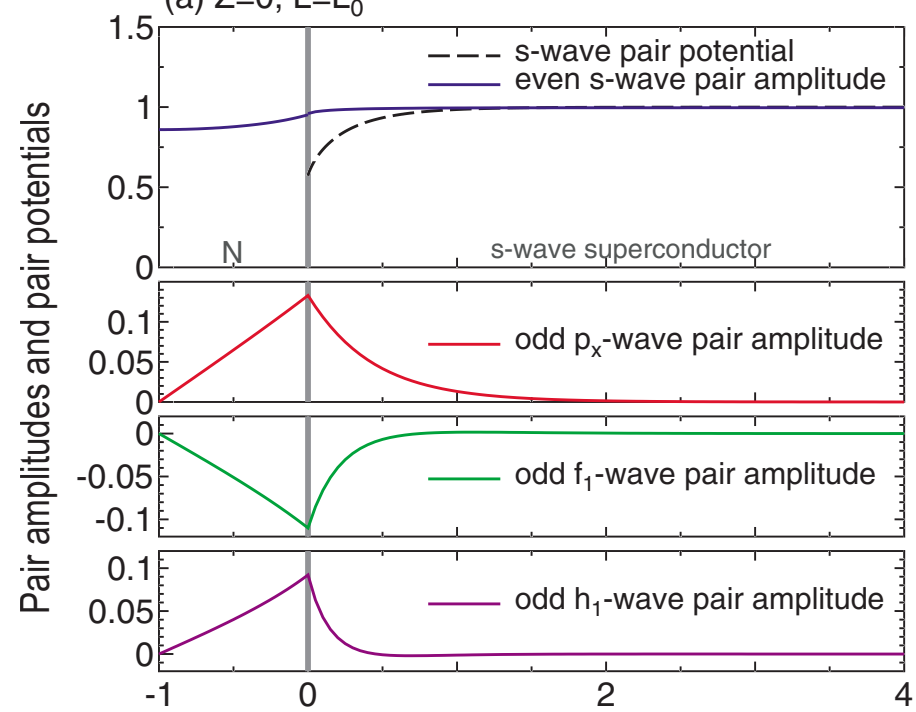

(c) $Z=0, L=5 L_{0}$

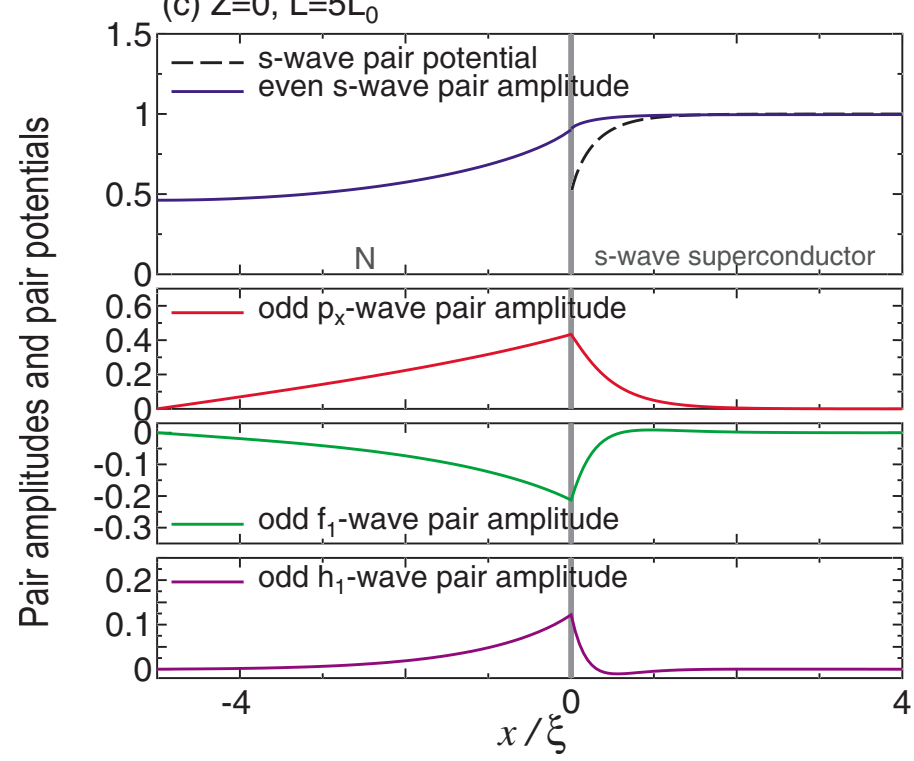

(b) $\mathrm{Z}=5, \mathrm{~L}=\mathrm{L}_{0}$

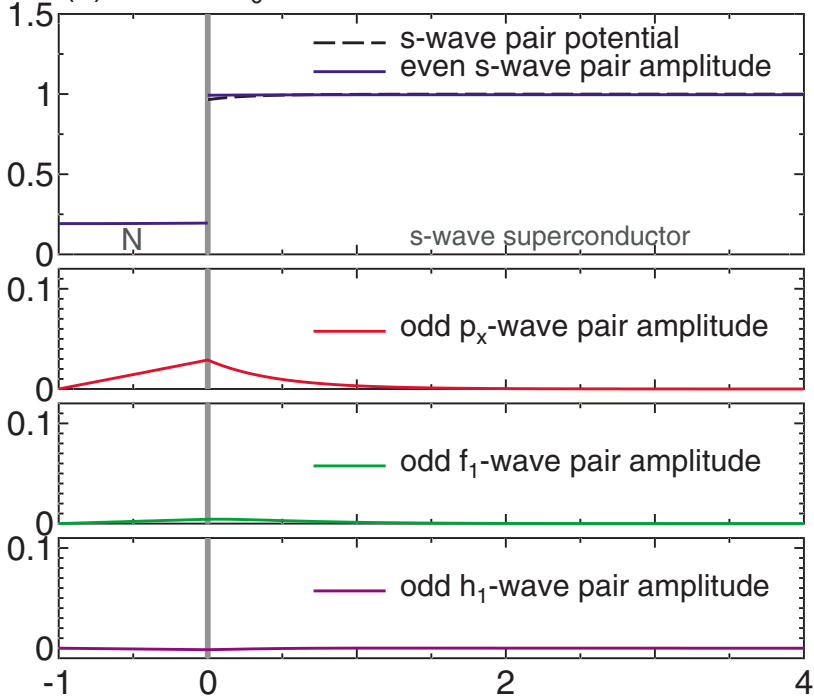

(d) $Z=5, L=5 L_{0}$
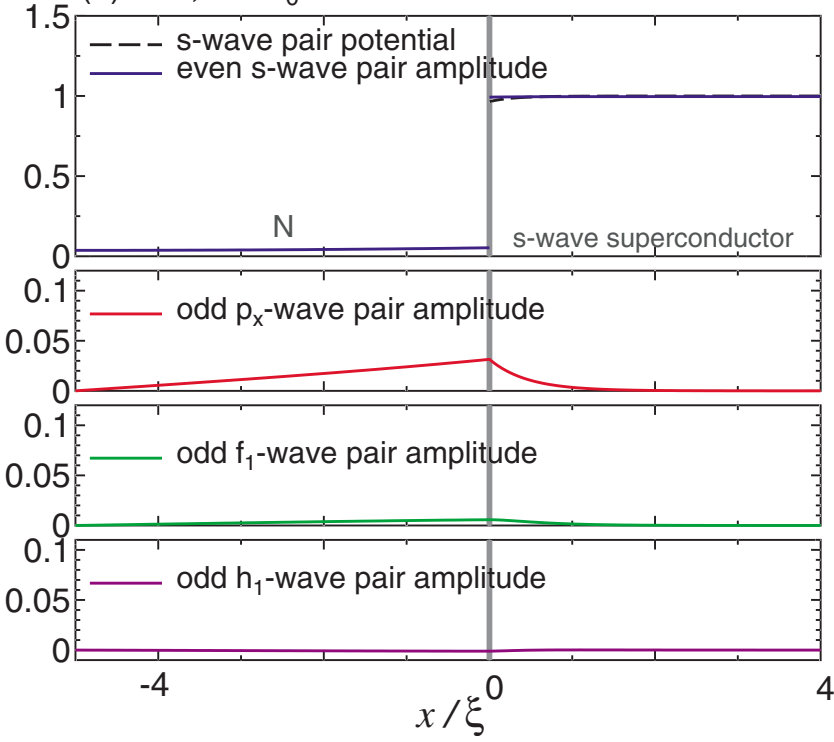

FIG. 1. (Color online) Spatial dependence of the normalized pair potential, even-frequency pair amplitude, and odd-frequency components of the pair amplitude for $s$-wave superconductor junctions. Here, we choose $\xi=v_{F} / \Delta_{0}$ in the $\mathrm{S}$ region $(x>0)$ and $\xi=L_{0}=v_{F} / 2 \pi T_{C}$ in the $\mathrm{N}$ region. The pair amplitudes $C_{0}^{(2)}, C_{1}^{(1)}, C_{3}^{(1)}$, and $C_{5}^{(1)}$ are denoted as even $s$-wave, odd $p_{x}$-wave, odd $f_{1}$-wave, and odd $h_{1}$-wave pair amplitudes. (a) $Z=0, L=L_{0}$, (b) $Z=5, L=L_{0}$, (c) $Z=0, L=5 L_{0}$, and (d) $Z=5, L=5 L_{0}$, respectively.

dependence of the pair potential and the pair amplitudes in the Matsubara frequency representation. We only concentrate on the lowest angular momentum of the even-frequency pair amplitude $C_{0}^{(2)}$. As regards the odd-frequency pair amplitudes, we focus on the $C_{1}^{(1)}, C_{3}^{(1)}$, and $C_{5}^{(1)}$ components, which all have odd parity and depend on $\theta$ as $\cos \theta, \cos 3 \theta$, and $\cos 5 \theta$, respectively, corresponding to the $p_{x}$-wave, $f_{1}$-wave, and $h_{1}$-wave components shown in Fig. 1 . In all cases, the even-frequency component is constant in the $\mathrm{S}$ region far away from the interface and the corresponding oddfrequency components are absent. The $s$-wave pair potential is suppressed for a full-transparency junction $(Z=0)$, while it is almost constant for a low-transparency one $(Z=5)$. It does not penetrate into the $\mathrm{N}$ region due to the absence of the attractive interaction in the $\mathrm{N}$ metal. On the other hand, in all considered cases, the spatial variation of the even-frequency $s$-wave pair amplitude is rather weak in the $\mathrm{S}$ region, while in the $\mathrm{N}$ region it is strong for $Z=0$ and is reduced for $Z$ $=5$ since the proximity effect is weaker in the latter case. The odd-frequency component always vanishes at $x=-L$ and does not have a jump at the N/S interface even for nonzero $Z$. Its amplitude is strongly enhanced near the N/S interface especially for full-transparency junctions. Note that not only the $p_{x}$ wave but also the $f_{1}$ wave and $h_{1}$ wave have sufficiently large magnitudes as shown in Figs. 1(a) and 1(c). With the decrease of the transparency of the N/S interface, the odd-frequency components are suppressed as shown in Figs. 1(b) and 1(d).

In order to understand the proximity effect in more detail, we look at the resulting LDOS and the spectral properties of 

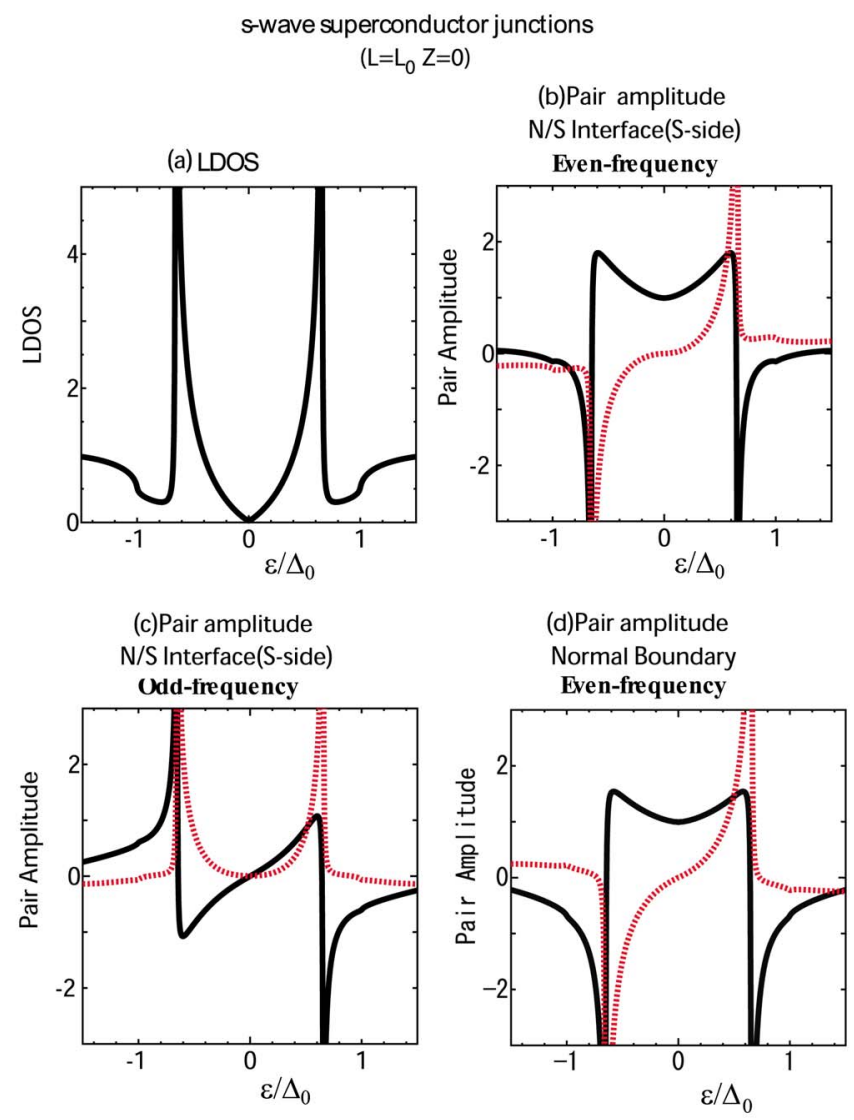

FIG. 2. (Color online) Energy dependence of the LDOS and the pair amplitudes in $s$-wave junctions with $L=L_{0}$ and $Z=0$. (a) The LDOS normalized by its value in the normal state. Solid line, LDOS on the S side of the N/S interface; dotted line, LDOS in the $\mathrm{N}$ region. Energy dependence of the real (solid line) and imaginary (dotted line) part of (b) even-frequency $s$-wave pair amplitude on the $\mathrm{S}$ side of the $\mathrm{N} / \mathrm{S}$ interface, (c) odd-frequency $p_{x}$-wave pair amplitude on the $\mathrm{S}$ side of the N/S interface, and (d) even-frequency $s$-wave pair amplitude at the edge of the $\mathrm{N}$ region.

pair amplitudes in real energy $\varepsilon$. We focus on the evenfrequency $s$-wave component of the pair amplitude $C_{0}^{(1)}$ and on odd-frequency $p_{x}$-wave pair amplitude $C_{1}^{(1)}$ on the $\mathrm{S}$ side of the N/S interface and on the edge of the $\mathrm{N}$ region. As follows from Eq. (14), the LDOS is independent of the coordinate $x$ in the $\mathrm{N}$ region. For $Z=0$ and $L=L_{0}$, the LDOS has a V-shaped structure. There is no jump of the value of the LDOS at the N/S interface. The even-frequency pair amplitude at the N/S boundary on the $\mathrm{S}$ side is shown in Fig. 2(b). Its real part is an even function of $\varepsilon$ while its imaginary part is an odd function of $\varepsilon$. The corresponding odd-frequency one is plotted in Fig. 2(c). In contrast to the even-frequency component [Fig. 2(b)], the real (imaginary) part of the pair amplitude is an odd (even) function of $\varepsilon$. The pair amplitude is enhanced around $\varepsilon \sim \pm 0.6 \Delta_{0}$ where the LDOS has a peak. At the N/S boundary $(x=-L)$, only the even-frequency component exists. The line shape of the pair amplitude shown in Fig. 2(d) is similar to that in Fig. 2(b).

In Fig. 3, the corresponding plots for $L=L_{0}$ and $Z=5$ are shown. The LDOS on the $\mathrm{S}$ side of the N/S interface has a U-shaped DOS similar to the bulk DOS. On the other hand,

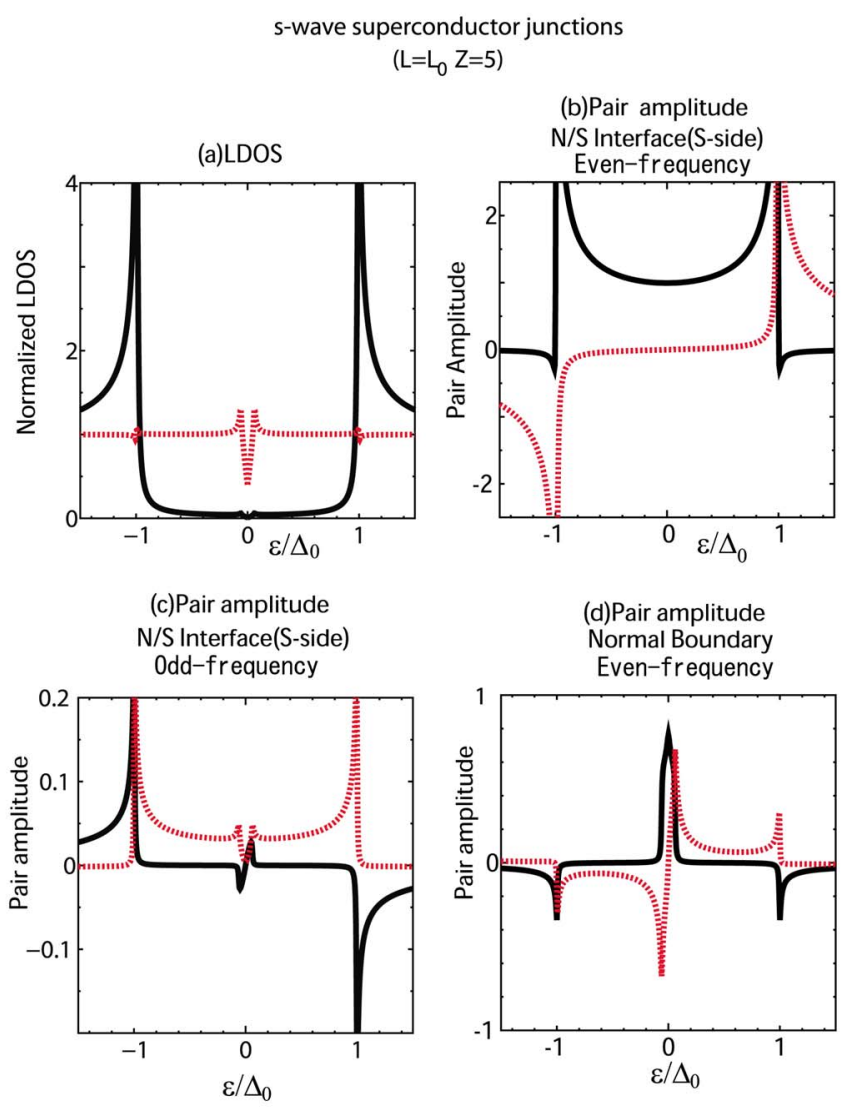

FIG. 3. (Color online) Same as Fig. 2, but with $L=L_{0}$ and $Z=5$.

in the $\mathrm{N}$ region, the LDOS has a different value due to the discontinuity at the N/S interface. The LDOS in N also exhibits a minigap structure which scales with the interface transparency, in accordance with the well-known McMillan model of proximity effect in conventional superconducting $\mathrm{N} / \mathrm{S}$ junctions. ${ }^{25}$ The magnitude of the real part of the evenfrequency component on the $S$ side of the N/S interface exceeds the magnitude of the imaginary part as seen from Fig. 3(b) for $|\varepsilon|<\Delta_{0}$. The magnitude of the odd-frequency part is small as compared to that of the even-frequency one. As seen from Fig. 3(c), the real part of the odd-frequency component has a minigap structure and the imaginary part has a dip and peak structure, in contrast to the case of the even-frequency one [see Fig. 3(b)]. At the N/S boundary $(x=-L)$, only the even-frequency component exists. The real part of the evenfrequency component at $x=-L$ has a peak around $\varepsilon=0$ [see Fig. 3(d)]. The width of this peak is of the same order as the dip of the LDOS. As compared to the corresponding case of $Z=0$, the proximity effect in the $\mathrm{N}$ region is essential only at low energy $\varepsilon$.

It is also interesting to consider the case of large width of the $\mathrm{N}$ region. Here, we concentrate on the situation with a full-transparency $\mathrm{N} / \mathrm{S}$ interface $(Z=0)$ and $L=5 L_{0}$. In this case the LDOSs in the $\mathrm{N}$ region and at the N/S interface coincide with each other as seen from Fig. 4. The LDOS has multiple peaks due to the existence of the multiple subgap structures due to electron-hole interference effects in the $\mathrm{N}$ region. ${ }^{26}$ The amplitudes of the corresponding even- 
s-wave superconductor junctions

$\left(\mathrm{L}=5 \mathrm{~L}_{0} \mathrm{Z}=0\right)$ (a) LDOS

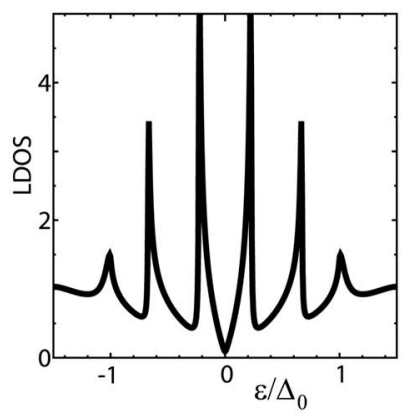

(c) Pair amplitude $\mathrm{N} / \mathrm{S}$ Interface(S-side) Odd-frequency

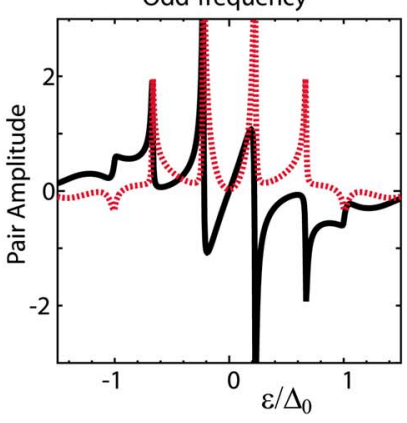

(b)Pair amplitude

N/S Interface(S-side)
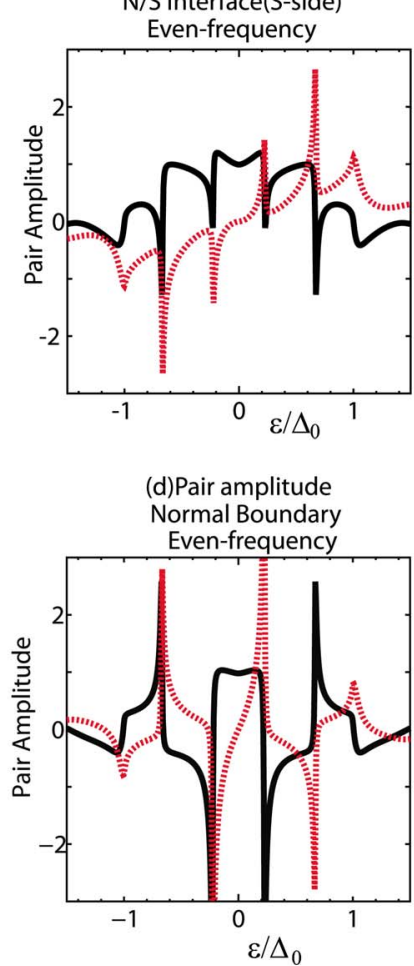

FIG. 4. (Color online) Same as Fig. 2, but with $L=5 L_{0}$ and $Z$ $=0$.

frequency and odd-frequency components are enhanced at energies $\varepsilon$ corresponding to the LDOS peak positions, while the ratio of these components depends on energy and location in the $\mathrm{N}$ region. To clarify this point much more clearly, we concentrate on the ratio of the odd- and even-frequency components in the $\mathrm{N}$ region. According to Eq. (13), the ratio of the magnitude of the odd-frequency component $f_{1+}^{(N)}(\varepsilon, \theta)$ to the even-frequency one $f_{2+}^{(N)}(\varepsilon, \theta)$ is

$$
\frac{\left|f_{1+}^{(N)}(\varepsilon, \theta)\right|}{\left|f_{2+}^{(N)}(\varepsilon, \theta)\right|}=\frac{|1 / t-t|}{|1 / t+t|}=\left|\tan \left(\frac{2 \varepsilon}{v_{F x}}(L+x)\right)\right| \text {. }
$$

At the edge of the $\mathrm{N}$ region, $x=-L$, the odd-frequency component vanishes at all energies. On the other hand, a very interesting situation occurs at the N/S interface, $x=0$, as will be shown below. In Fig. 5, we plot this ratio for $\theta=0$ and $x=0$. It is remarkable that at some energies the amplitude of the odd-frequency pair exceeds that of the even-frequency one.

Let us clarify the relation between the positions of the bound states and the above ratio of the odd-to-even pair amplitude. In the limit $L \gg L_{0}$ the bound states are determined by a simple relation for $Z=0,{ }^{26}$

$$
\varepsilon_{n}=\frac{\pi v_{F x}}{2 L}(n+1 / 2), \quad n=0,1,2, \ldots
$$

A very dramatic situation occurs at the N/S interface, $x=0$ : combining the above two equations, we obtain that at the

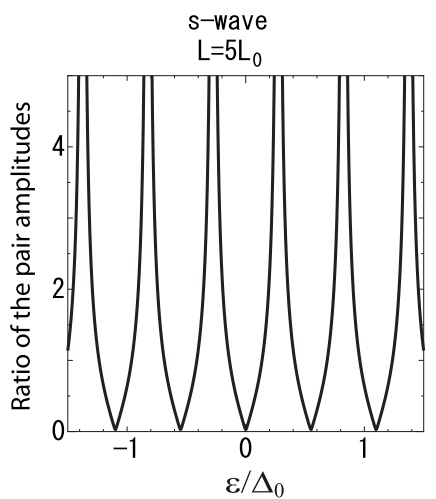

FIG. 5. Ratio of the pair amplitudes $f_{1+}^{(N)}(\varepsilon, \theta) / f_{2+}^{(N)}(\varepsilon, \theta)$ on the $\mathrm{N}$ side of the N/S interface in $s$-wave junction as a function of energy $\varepsilon$ for $\theta=0$ and $L=5 L_{0}$.

LDOS peak positions $\varepsilon=\varepsilon_{n}$ the ratio of the odd-to-even pair amplitude diverges,

$$
\frac{\left|f_{1+}^{(N)}(\varepsilon, \theta)\right|}{\left|f_{2+}^{(N)}(\varepsilon, \theta)\right|}=|\tan (\pi / 2+\pi n)|=\infty .
$$

That means that at the subgap peak energies the oddfrequency component dominates over the even-frequency one at the N/S interface. This is a remarkable property of the odd-frequency pairing, which makes it relevant to the classical McMillan-Rowell oscillations in the N/S geometry. ${ }^{26}$ To summarize, we have shown that the odd-frequency component is present even in the standard case of a ballistic N/S system, and it dominates at energies when the LDOS has subgap peaks.

\section{B. $p_{x}$-wave pair potential}

Next, we focus on the $p_{x}$-wave superconductor junctions as shown in Fig. 6. As in the case of $s$-wave junctions, by changing the length of the normal region $L$ and the transparency at the interface, we calculate the spatial dependence of the pair potential and the pair amplitudes in the Matsubara frequency representation. We concentrate only on the lowest angular momentum of the even-frequency pair amplitude $C_{1}^{(2)}$. As regards the odd-frequency pair amplitude, we focus on the $C_{0}^{(1)}, C_{2}^{(1)}$, and $C_{4}^{(1)}$ components where the parity of the odd-frequency components is even. These functions corre-

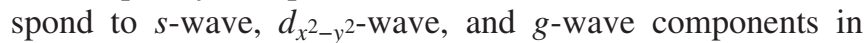
Fig. 6, where the $\theta$ dependencies are given by $1, \cos 2 \theta$, and $\cos 4 \theta$, respectively. In all cases, the even-frequency component is constant in the $\mathrm{S}$ region far away from the interface and the corresponding odd-frequency components are absent. The $p_{x}$-wave pair potential is reduced at the N/S interface in all cases. For $Z=5$, the reduction is significant and the resulting magnitude of the $p_{x}$-wave pair potential is almost zero at the N/S interface. It does not penetrate into the $\mathrm{N}$ region due to the absence of the attractive interaction in the $\mathrm{N}$ metal. The amplitude of the $p_{x}$-wave even-frequency pair amplitude is reduced toward the N/S interface and monotonically decreases in the $\mathrm{N}$ region. It does not have a jump at the $\mathrm{N} / \mathrm{S}$ interface even for nonzero $Z$ and vanishes at the edge of the 
(a) $\mathrm{Z}=0, \mathrm{~L}=\mathrm{L}_{0}$

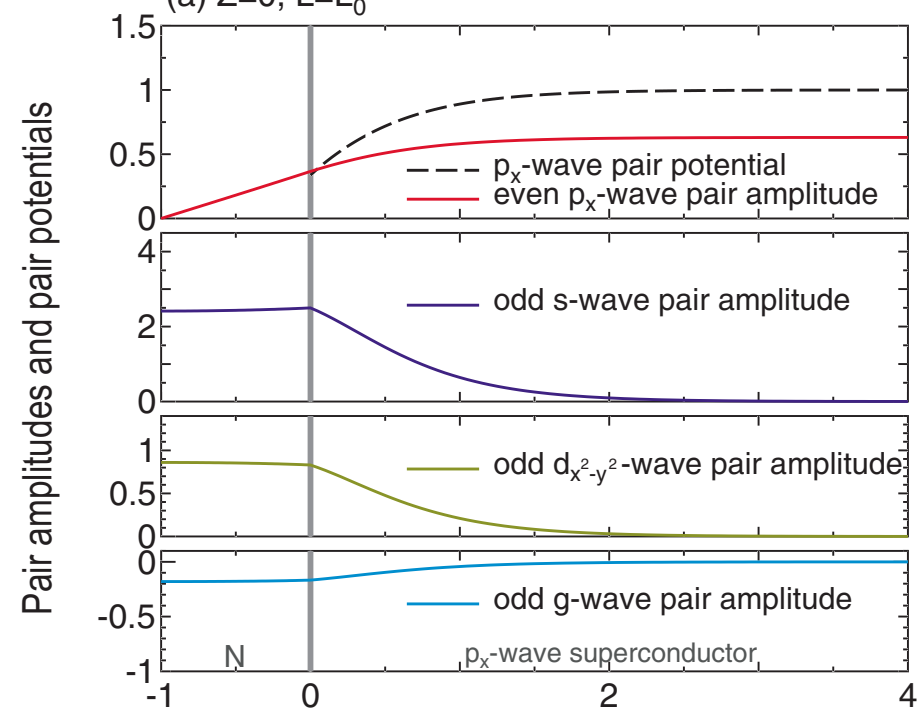

(c) $Z=0, L=5 L_{0}$

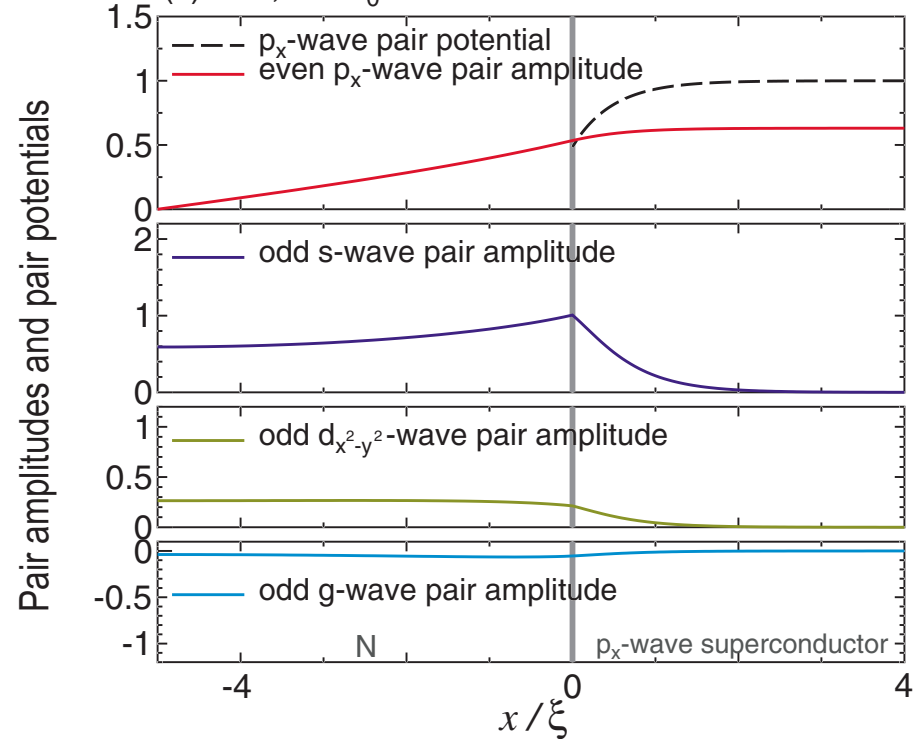

(b) $Z=5, L=L_{0}$

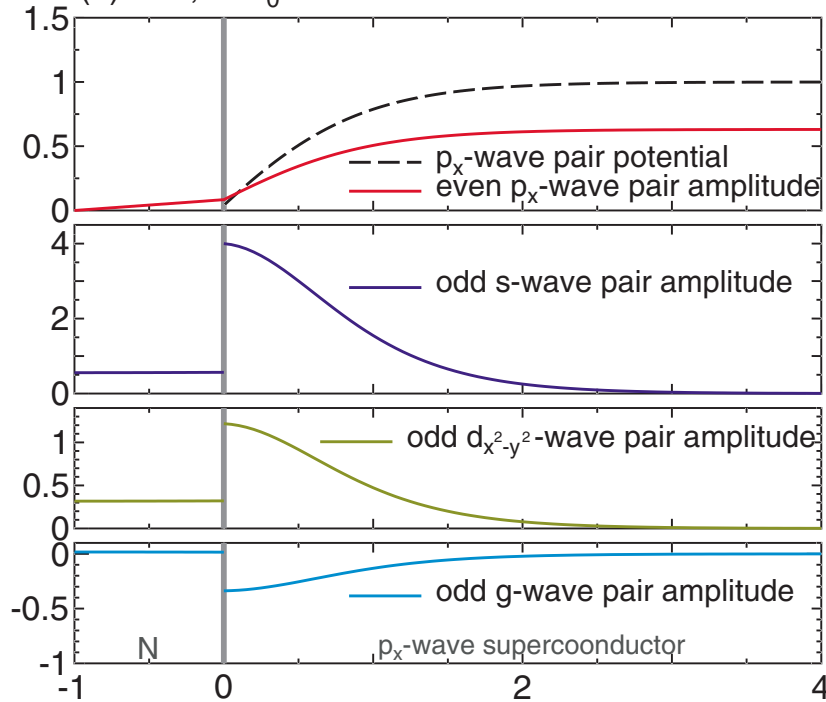

(d) $Z=5, L=5 L_{0}$

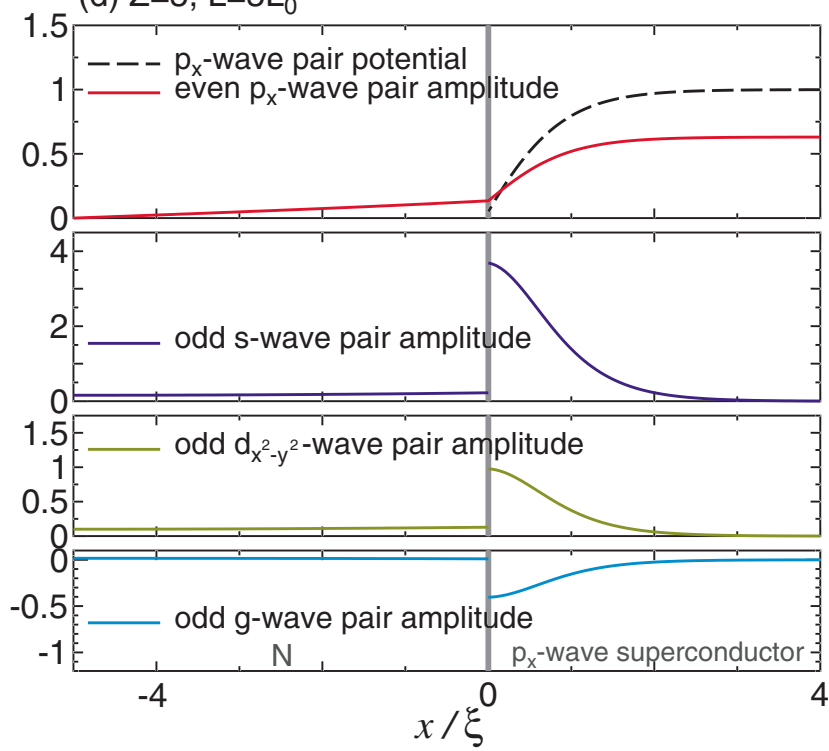

FIG. 6. (Color online) Spatial dependence of the normalized pair potential and even-frequency and odd-frequency pair amplitudes for $p_{x}$-wave superconductor junctions. Here, we choose $\xi=v_{F} / \Delta_{0}$ in the $\mathrm{S}$ region $(x>0)$ and $\xi=L_{0}=v_{F} / 2 \pi T_{C}$ in the $\mathrm{N}$ region. The pair

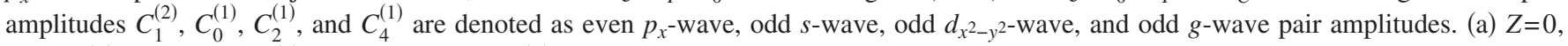
$L=L_{0}$, (b) $Z=5, L=L_{0}$, (c) $Z=0, L=5 L_{0}$, and (d) $Z=5, L=5 L_{0}$, respectively.

$\mathrm{N}$ region $(x=-L)$. On the other hand, the odd-frequency component is always nonzero at $x=-L$ and has a jump at the N/S interface for nonzero $Z$ [see Figs. 6(b) and 6(d)]. The amplitude of the odd-frequency component is strongly enhanced near the $\mathrm{S}$ side of the N/S interface. This enhancement is much more significant for the low-transparency interface with large $Z$ [see Figs. 6(b) and 6(d)]. However, for the presently chosen Matsubara frequency $\omega_{n}=0.05 \pi T_{C}$ it cannot penetrate into the $\mathrm{N}$ region. On the other hand, for $Z=0$, the odd-frequency component significantly extends into the $\mathrm{N}$ region. Note that not only the $s$-wave but also the $d_{x^{2}-y^{2}}$-wave and $g$-wave components have sufficiently large magnitudes as shown in Figs. 6(a) and 6(c). These pair amplitudes are almost constant in the $\mathrm{N}$ region.

In order to get better insight into the spectral property of the odd-frequency pair amplitude, we calculate the LDOS and the pair amplitudes as functions of real energy $\varepsilon$. We focus on the even-frequency $p_{x}$-wave component of the pair amplitude $C_{1}^{(2)}$ and odd-frequency $s$-wave component of the pair amplitude $C_{0}^{(1)}$ at the $\mathrm{N} / \mathrm{S}$ interface on the S side and the $\mathrm{N}$ boundary. In the $\mathrm{N}$ region, the LDOS is independent of $x$ as shown by Eq. (14). For $Z=0$ and $L=L_{0}$ [see Fig. 7(a)], the LDOS has a zero-energy peak (ZEP) due to the formation of the MARS. There is no jump of the LDOS at the interface due to the full transparency. The even-frequency pair amplitude at the $\mathrm{S}$ side of the N/S boundary is shown in Fig. 7(b). Neither the real nor imaginary part varies strongly around $\varepsilon \sim 0$.

Similar to the case of the $s$-wave junctions, the real part of the even-frequency component is an even function of $\varepsilon$ while the imaginary part is an odd function of $\varepsilon$. The corresponding odd-frequency component is plotted in Fig. 7(c). The real 

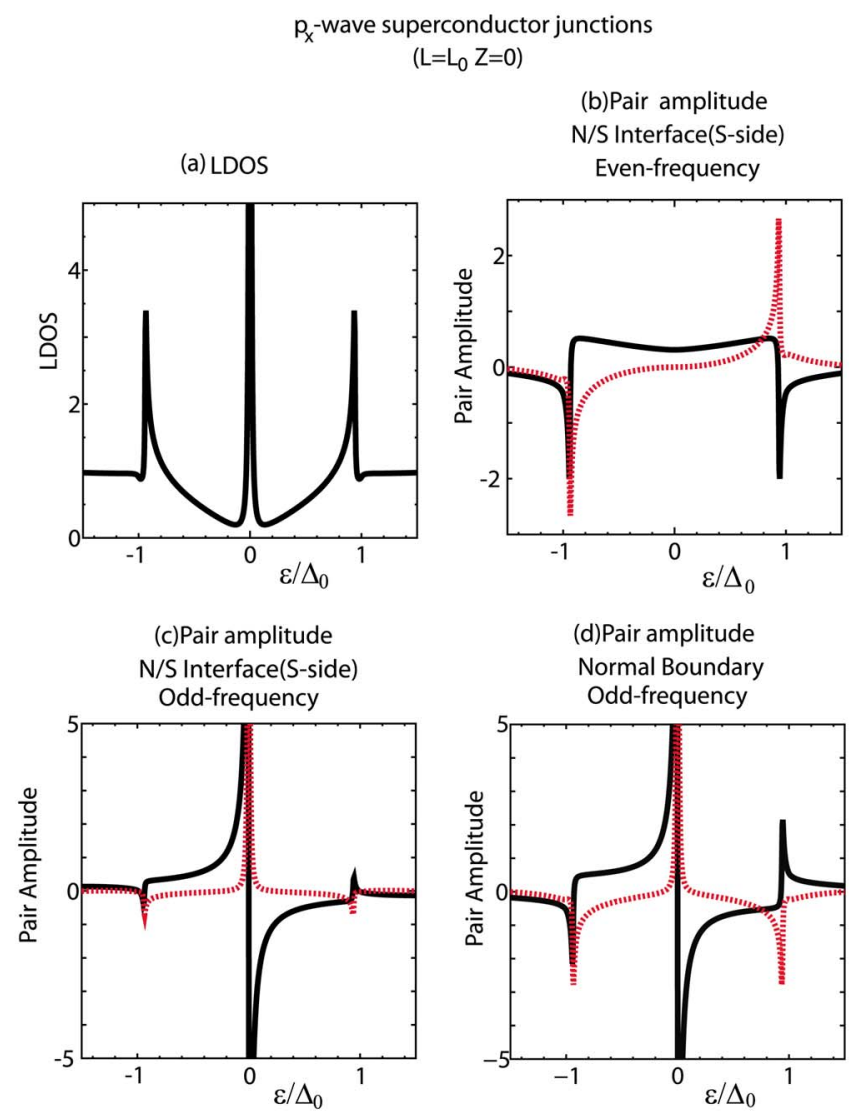

FIG. 7. (Color online) Energy dependence of the LDOS and the pair amplitudes in $p_{x}$-wave junctions with $L=L_{0}$ and $Z=0$. (a) The LDOS normalized by its value in the normal state. Solid line, LDOS on the $\mathrm{S}$ side of the N/S interface; dotted line, LDOS in the $\mathrm{N}$ region. Energy dependence of the real (solid line) and the imaginary (dotted line) part of (b) even-frequency $p_{x}$-wave pair amplitude on the $\mathrm{S}$ side of the N/S interface, (c) odd-frequency $s$-wave pair amplitude on the $\mathrm{S}$ side of the N/S interface, and (d) odd-frequency $s$-wave pair amplitude at the edge of the $\mathrm{N}$ region.

(imaginary) part of the pair amplitude is an odd (even) function of $\varepsilon$. The amplitude of the odd-frequency pair amplitude is enhanced around $\varepsilon=0$ where the LDOS has a ZEP. At the edge of the $\mathrm{N}(x=-L)$, only the odd-frequency component exists. The line shape of the pair amplitude shown in Fig. 7(d) is similar to that shown in Fig. 7(c).

In Fig. 8, the results of the corresponding calculation with $L=L_{0}$ and $Z=5$ are shown. Both the LDOS at the N/S interface and the edge of the $\mathrm{N}$ have a ZEP. In the $\mathrm{N}$ region, the LDOS is almost unity due to the absence of the proximity effect for $|\varepsilon|>0.24 \Delta_{0}$ [see dotted line in Fig. 8(a)]. The LDOS has a ZEP and small peak at $\varepsilon=0.24 \Delta_{0}$. The corresponding real and imaginary parts of the even-frequency pair amplitude at the N/S interface also have peaks at this energy [Fig. 8(b)]. The amplitude of the odd-frequency component is enhanced as compared to the corresponding evenfrequency one as shown in Fig. 8(c). At the edge of the $\mathrm{N}$ region $(x=-L)$, the amplitude of the odd-frequency component is almost zero for $|\varepsilon|>0.24 \Delta_{0}$. However, around zero energy, the amplitude of the odd-frequency component is drastically enhanced as in the case of $S$ side of the N/S
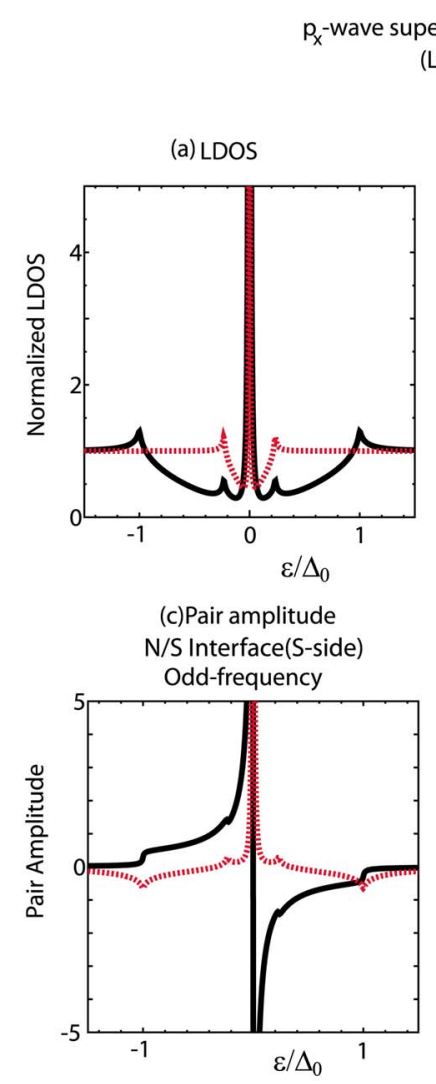
erconductor junctions $\left(\mathrm{L}=\mathrm{L}_{0} \mathrm{Z}=5\right)$

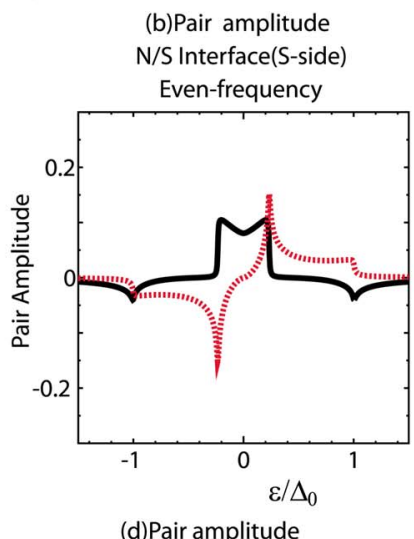

(d)Pair amplitude Normal Boundary Odd-frequency

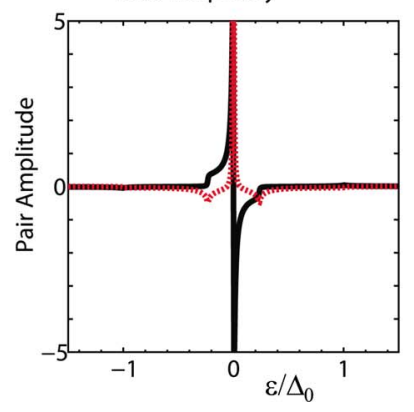

FIG. 8. (Color online) Same as Fig. 7, but with $L=L_{0}$ and $Z=5$.

boundary. The penetration of the odd-frequency component occurs only at low energies.

For the longer normal region with $L=5 L_{0}$, the resulting LDOS has a ZEP and a number of peaks at finite energies $\varepsilon$ [see Fig. 9(a)]. The even-frequency component of the pair amplitude on the $\mathrm{S}$ side of the N/S boundary also has multiple peaks. The corresponding odd-frequency component has many peaks with amplitudes strongly enhanced around $\varepsilon=0$. Around zero energy, the amplitude of the oddfrequency component is much larger than that of the evenfrequency one [see Fig. 9(c)]. At the edge of the $\mathrm{N}$ region, the resulting odd-frequency component has a significant amplitude as shown in Fig. 9(d). Finally, we focus on the ratio of the odd- and even-frequency components of the pair amplitude, $f_{1+}^{(N)}(\varepsilon, \theta) / f_{2+}^{(N)}(\varepsilon, \theta)$. In Fig. 10, we plot this ratio for $\theta=0$ and $x=0$. Remarkably, at some energies the oddfrequency pair amplitude exceeds that of the even-frequency one. In contrast to the $s$-wave case, there is a huge peak at $\varepsilon=0$ corresponding to the existence of the MARS.

To summarize, we have shown that when the LDOS has a ZEP, the resulting odd-frequency component is enhanced around $\varepsilon=0$, its imaginary part having a ZEP. It is evident that the odd-frequency pairing state is indispensable in understanding the proximity effect in a $p_{x}$-wave superconductor system.

\section{C. $d_{x y}$-wave pair potential}

Finally we focus on the $d_{x y}$-wave junctions as shown in Fig. 11. Similar to the above two cases, by changing the 


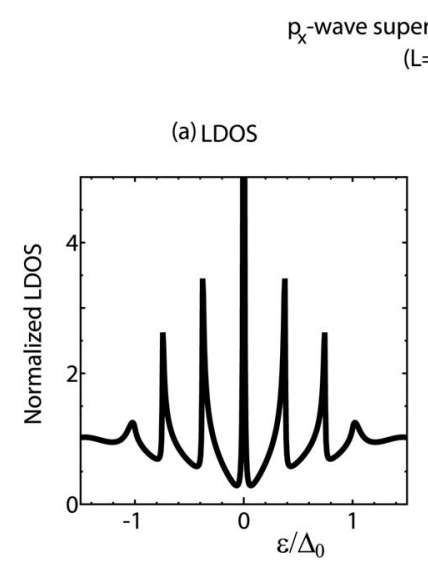

$\left(\mathrm{L}=5 \mathrm{~L}_{0} \mathrm{Z}=0\right)$

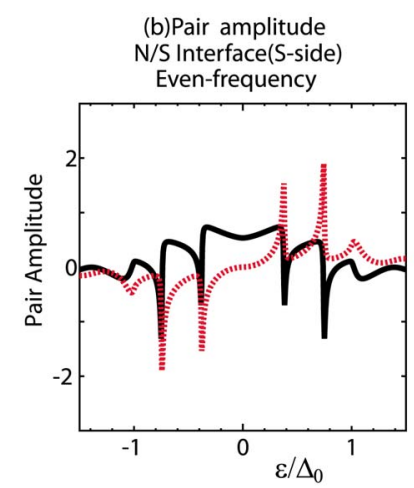

(c)Pair amplitude $\mathrm{N} / \mathrm{S}$ Interface(S-side) Odd Component
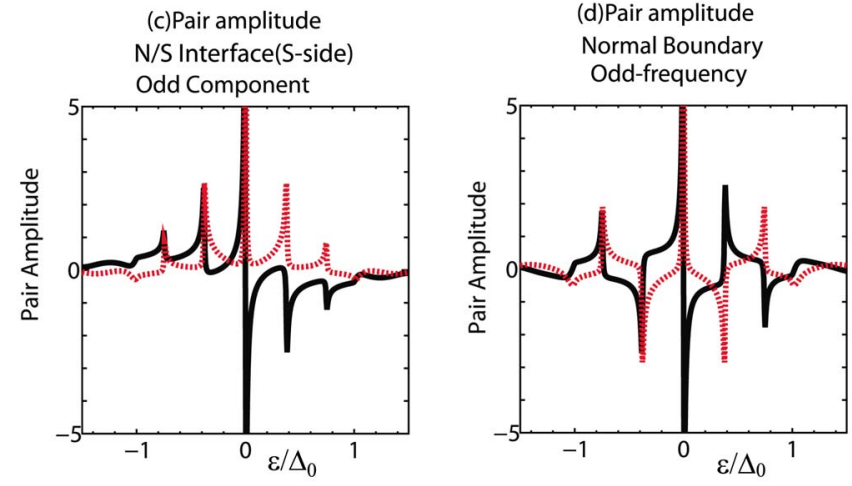

FIG. 9. (Color online) Same as Fig. 8, but with $L=5 L_{0}$ and $Z$ $=0$.

length of the normal region $L$ and the transparency at the interface, we calculate the spatial dependence of the pair potential and the pair amplitudes in the Matsubara frequency representation. Here we only concentrate on the lowest angular momentum of the even-frequency pair amplitude $S_{2}^{(2)}$. The $s$-wave component of the pairing amplitude is absent due to the sign change of the pair potential with respect to the exchange $\theta$ by $-\theta$. As regards the odd-frequency pair amplitude, we focus on the $S_{1}^{(1)}, S_{3}^{(1)}$, and $S_{5}^{(1)}$ components where the spatial parity of the odd-frequency components is odd. These cases correspond to the $p_{y}$-wave, $f_{2}$-wave, and $h_{2}$-wave components in Fig. 11, where the $\theta$ dependence is given by $\sin \theta, \sin 3 \theta$, and $\sin 5 \theta$, respectively. In all cases, the even-frequency component is constant in the $\mathrm{S}$ region far away from the interface and the corresponding oddfrequency components are absent. The $d_{x y}$-wave pair potential is suppressed at the N/S interface in all cases. For $Z=5$, the reduction is significant and it is almost zero at the N/S interface. The even-frequency $d_{x y}$-wave pair amplitude is reduced toward the N/S interface and monotonically decreases in the $\mathrm{N}$ region similar to the case of the $p_{x}$-wave one [see Figs. 6(a) and 11(a)]. It does not have a jump at the N/S interface even for nonzero $Z$ and vanishes at the edge of $\mathrm{N}$ region $(x=-L)$. On the other hand, the odd-frequency component is always nonzero at $x=-L$ and has a jump at the N/S interface. The amplitude of the odd-frequency component is strongly enhanced near the $\mathrm{S}$ side of the N/S interface. This enhancement is much more significant for the lowtransparency interface with large magnitude of $Z$ [see Figs.

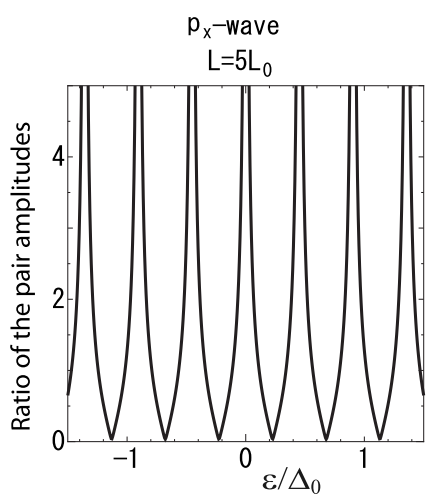

FIG. 10. Ratio of the pair amplitudes $f_{1+}^{(N)}(\varepsilon, \theta) / f_{2+}^{(N)}(\varepsilon, \theta)$ as a function of $\varepsilon$ for $L=5 L_{0}$ at the $\mathrm{N}$ side of the N/S interface for $p_{x}$-wave junctions for $\theta=0$.

11(b) and $11(\mathrm{~d})]$. On the other hand, for $Z=0$, the oddfrequency components significantly penetrate into the $\mathrm{N}$ region. Note that not only the $p_{y}$-wave but also $f_{2}$-wave and $h_{2}$-wave components have sufficiently large magnitudes as shown in Figs. 11(a) and 11(c). The above pair amplitudes are almost constant in the $\mathrm{N}$ region.

In order to get better insight into the spectral property of the odd-frequency pair amplitude, we calculate the LDOS and the pair amplitudes as functions of real energy $\varepsilon$. We focus on the even-frequency $d_{x y}$-wave component of the pair amplitude $S_{2}^{(2)}$ and odd-frequency $p_{y}$-wave component of the pair amplitude $S_{1}^{(1)}$ at the $S$ side of the N/S interface and at the edge of the $\mathrm{N}$ region, $x=-L$. The resulting LDOS has a ZEP due to the formation of the MARS. As in the previously considered cases of $s$-wave and $p_{x}$-wave junctions, the LDOS is independent of $x$ as follows from Eq. (14). Here, we choose $Z=0$ and $L=L_{0}$ [see Fig. 12(a)]. Similar to the $p_{x}$-wave case, the even-frequency pair amplitude at the N/S boundary on the $\mathrm{S}$ side is shown in Fig. 12(b). Neither the real nor imaginary part varies strongly around $\varepsilon \sim 0$. The real part of the even-frequency component is an even function of $\varepsilon$ while its imaginary part is an odd function of $\varepsilon$. The corresponding odd-frequency component is plotted in Fig. 12(c). In contrast to the even-frequency component [Fig. 12(b)], the real (imaginary) part of the pair amplitude is an odd (even) function of $\varepsilon$. The amplitude of the pair amplitude is enhanced around $\varepsilon \sim 0$ where the LDOS has a ZEP. At the N/S boundary $(x=-L)$, only the odd-frequency component exists. The line shape of the pair amplitude, as shown in Fig. 12(d), is qualitatively similar to that in Fig. 12(c). This qualitative behavior of the line shapes is very similar to that for the corresponding $p_{x}$-wave case.

Similar to the $p_{x}$-wave junction case, when the LDOS has a ZEP, the resulting odd-frequency component is enhanced around $\varepsilon=0$. It is evident that the odd-frequency pairing state is indispensable in understanding the proximity effect in a $d_{x y}$-wave superconductor system.

In the following, we comment on the differences between odd-frequency pair amplitudes in $d_{x y}$-wave and $p_{x}$-wavejunctions. In both cases, the magnitude of the oddfrequency component is enhanced at the interface and in the normal region. However, the odd-frequency odd-parity state 
(a) $Z=0, L=L_{0}$

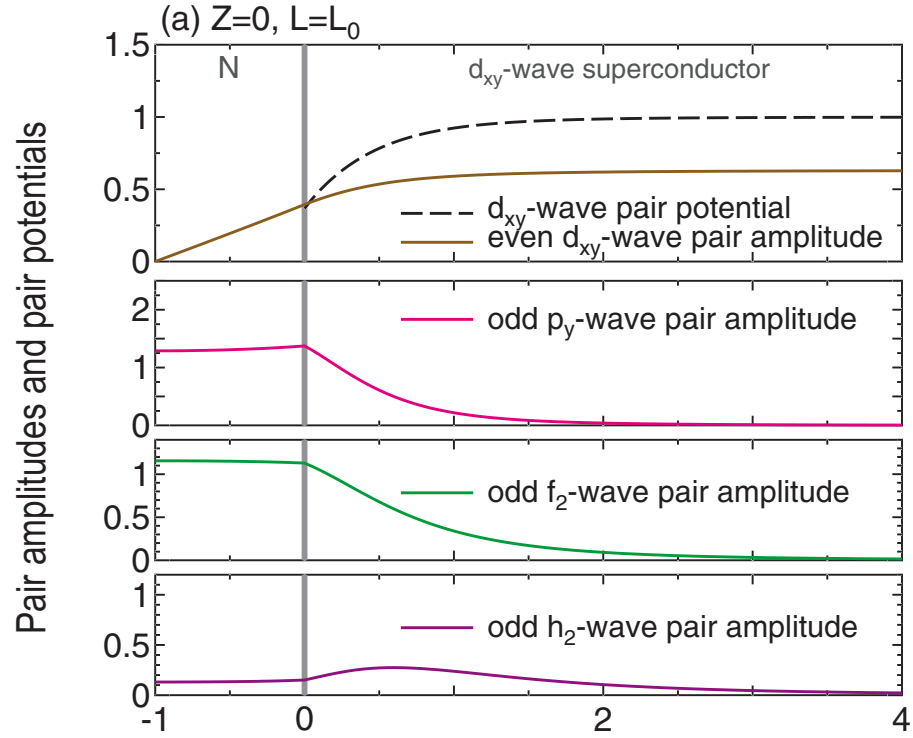

(c) $Z=0, L=5 L_{0}$

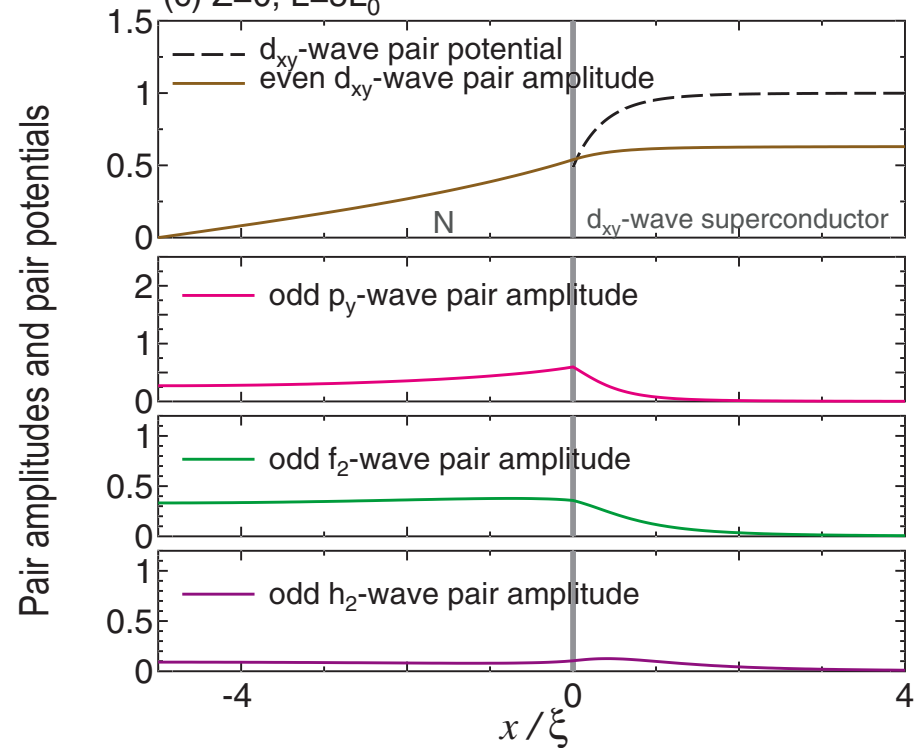

(b) $Z=5, L=L_{0}$
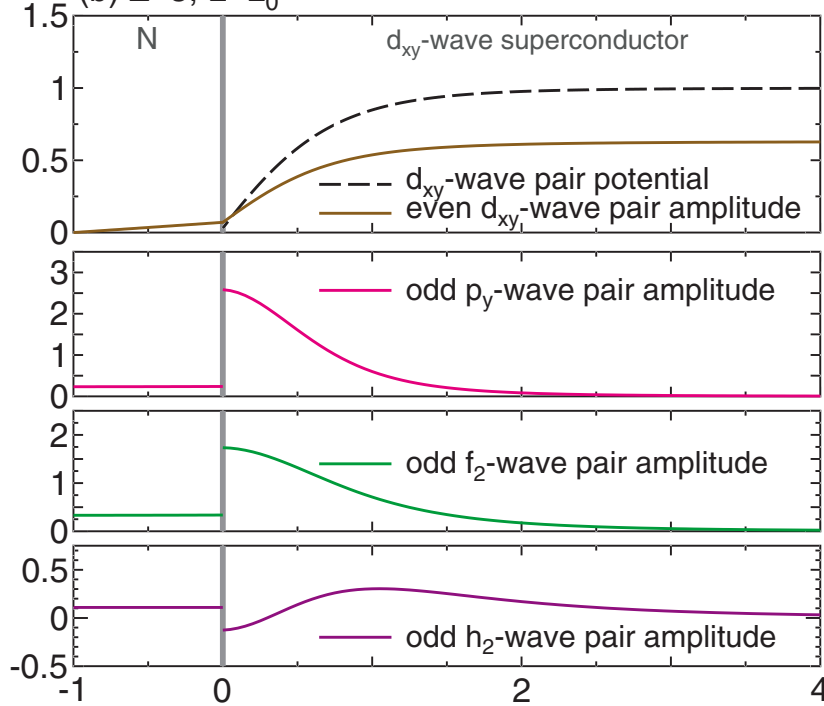

(d) $Z=5, L=5 L_{0}$
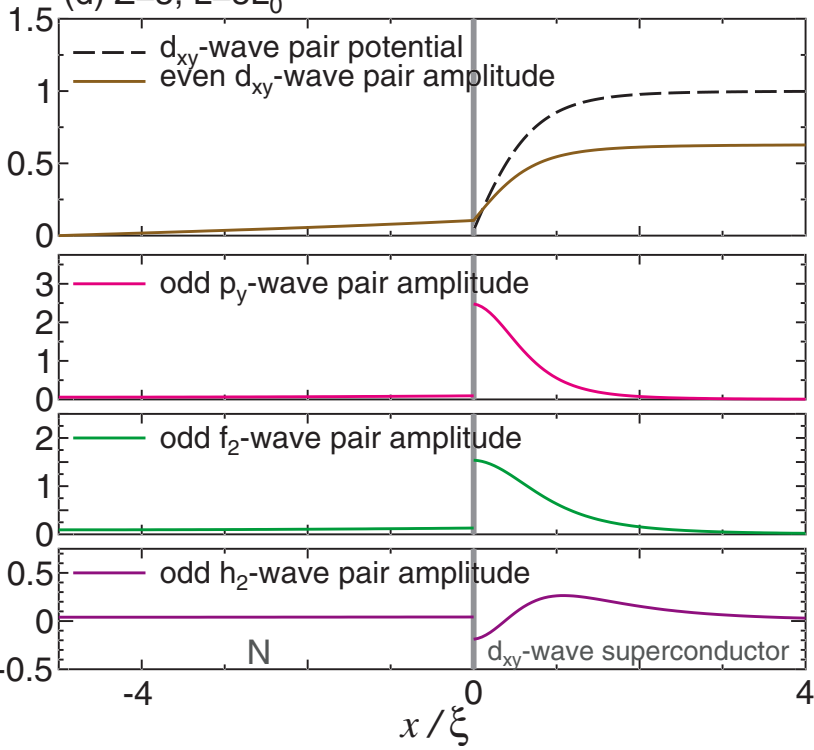

FIG. 11. (Color online) Spatial dependence of the normalized pair potential and even-frequency and odd-frequency pair amplitudes for $d_{x y}$-wave superconductor junctions. Here, we choose $\xi=v_{F} / \Delta_{0}$ in the $\mathrm{S}$ region $(x>0)$ and $\xi=L_{0}=v_{F} / 2 \pi T_{C}$ in the $\mathrm{N}$ region. The pair amplitudes $S_{2}^{(2)}, S_{1}^{(1)}, S_{3}^{(1)}$, and $S_{5}^{(1)}$ are denoted as even $d_{x y}$-wave, odd $p_{y}$-wave, odd $f_{2}$-wave, and odd $h_{2}$-wave pair amplitudes. (a) $Z=0$, $L=L_{0}$, (b) $Z=5, L=L_{0}$, (c) $Z=0, L=5 L_{0}$, and (d) $Z=5, L=5 L_{0}$, respectively.

is generated for the $d_{x y}$-wave case, while the odd-frequency even-parity state is generated for the $p_{x}$-wave case. The $s$-wave isotropic component which is robust against the impurity scattering ${ }^{12}$ appears only in the latter case. Then the $d_{x y}$-wave pair amplitude cannot penetrate into diffusive normal metal while the $p_{x}$-wave one can. Thus we can naturally understand the presence of the proximity effect with the MARS in $p_{x}$-wave junctions ${ }^{12,13}$ and its absence in $d_{x y}$-wave junctions. ${ }^{12,27}$

It is instructive to relate the LDOS anomalies in $d_{x y}$-wave and $p_{x}$-wave junctions to the magnitude of the oddfrequencypairing component. According to Eq. (13), in $d_{x y^{-}}$ and $p_{x}$-wave junctions the ratio of the magnitude of the oddfrequency component $f_{1+}^{(N)}(\varepsilon, \theta)$ to the even-frequency one $f_{2+}^{(N)}(\varepsilon, \theta)$ is

$$
\frac{\left|f_{1+}^{(N)}(\varepsilon, \theta)\right|}{\left|f_{2+}^{(N)}(\varepsilon, \theta)\right|}=\frac{|1 / t+t|}{|1 / t-t|}=\left|\operatorname{cotan}\left(\frac{2 \varepsilon}{v_{F x}}(L+x)\right)\right|
$$

It follows from the above expression that at the edge of the $\mathrm{N}$ region, $x=-L$, the odd-frequency component dominates at all energies. On the other hand, at the N/S interface, $x=0$, the odd-frequency component dominates at energies $\varepsilon$ $=\varepsilon_{n}$ corresponding to the LDOS peak positions.

$$
\varepsilon_{n}=\frac{\pi v_{F x} n}{2 L}, \quad n=0,1,2, \ldots
$$

for $Z=0$ and $L \gg L_{0}$. For $n=0$ Eq. (34) describes the midgap Andreev bound state and higher $n$ correspond to the subgap resonances for large $\mathrm{N}$ region thickness. Therefore, we can 
$d_{x y}$-wave superconductor junctions $\left(\mathrm{L}=\mathrm{L}_{0} \mathrm{Z}=0\right)$ (a) LDOS

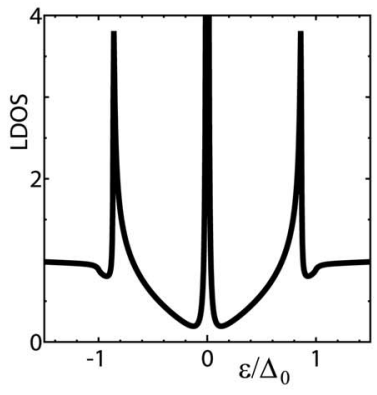

(c) Pair amplitude N/S Interface(S-side) Odd-frequency

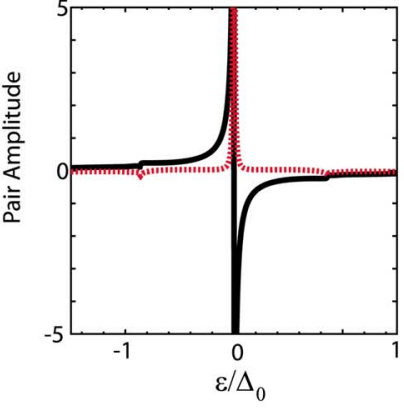

(b)Pair amplitude N/S Interface(S-side) Even-frequency

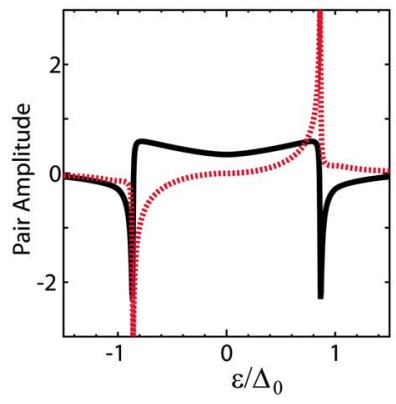

(d)Pair amplitude Normal Boundary Odd-frequency

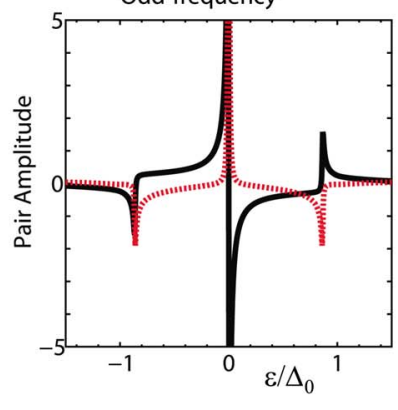

FIG. 12. (Color online) Energy dependence of the LDOS and the pair amplitudes in $d_{x y}$-wave junctions with $L=L_{0}$ and $Z=0$. (a) The LDOS normalized by its value in the normal state. Solid line, LDOS on the S side of the N/S interface; dotted line, LDOS in the $\mathrm{N}$ region. Energy dependence of the real (solid line) and the imaginary (dotted line) part of (b) even-frequency $d_{x y}$-wave pair amplitude on the $\mathrm{S}$ side of the N/S interface, (c) odd-frequency $p_{y}$-wave pair amplitude on the $\mathrm{S}$ side of the N/S interface, and (d) oddfrequency $p_{y}$-wave pair amplitude at the edge of the $\mathrm{N}$ region.

conclude that in $d_{x y}$-wave and $p_{x}$-wave junctions the oddfrequency component dominates over the even-frequency one at the N/S interface at the energies corresponding to the LDOS peak positions, including the prominent zero-energy peak. Moreover, the odd-frequency component always dominates at the edge of the $\mathrm{N}$ region, $x=-L$, where the breaking of translational invariance is strongest due to a sign change of the pair amplitude at that point.

\section{CONCLUSIONS}

In summary, using the quasiclassical Green's function formalism, we have shown that the odd-frequency pairing state is ubiquitously generated in the normal-metal/ superconductor ballistic junction system, where the length of the normal region is finite. It is shown that the even-parity (odd-parity) pair potential in the superconductor induces an odd-frequency pairing component with spin-singlet odd parity (spin-triplet even parity). As regards the symmetry of the superconductor, we have chosen typical three cases: spinsinglet $s$ wave, spin-triplet $p_{x}$ wave, and spin-singlet $d_{x y}$ wave. In the latter two cases, a midgap Andreev resonant state appears at the N/S interface. Even for conventional $s$-wave junctions, the amplitude of the odd-frequency pairing state is enhanced at the N/S interface with full transparency. By analyzing the spectral properties of the pair amplitudes, we found that the magnitude of the resulting odd-frequency component at the interface can exceed that of the evenfrequency one. For the case of $p_{x}$-wave and $d_{x y}$-wave junctions, the magnitude of the odd-frequency component at the $\mathrm{S}$ side of the N/S interface is significantly enhanced. The magnitude of the induced odd-frequency component is enhanced in the presence of the midgap Andreev resonant state due to the sign change of the anisotropic pair potential at the interface. The LDOS has a zero-energy peak both at the interface and in the $\mathrm{N}$ region. At the edge of the $\mathrm{N}$ region, only the odd-frequency component is nonzero.

The underlying physics behind these phenomena is related to the breakdown of translational invariance near the $\mathrm{N} / \mathrm{S}$ interface where the pair potential $\bar{\Delta}_{ \pm}(x)$ acquires a spatial dependence. As a result, an odd-frequency component is quite generally induced near the interface. The breakdown of translational invariance is the strongest when the pair potential changes sign upon reflection as in the case of $p_{x}$-wave and $d_{x y}$-wave junctions, then the magnitude of the oddfrequency component is the largest. Moreover, the phase of the interface-induced odd-frequency component has a $\pi / 2$ shift from that in the bulk of S. Therefore, as shown above, the odd-frequency component $f_{1 \pm}^{(i)}\left(\omega_{n}, \theta\right)$ becomes a purely imaginary number and the peak structure in the LDOS naturally follows from the normalization condition.

We have also shown that in the N/S junctions with $s$-wave superconductors the classical McMillan-Rowell oscillations $^{26}$ can also be reinterpreted in terms of odd-frequency pairing. As follows from Eq. (32), at the energies corresponding to the subgap peaks in the N/S junction, the oddfrequency component dominates over the even-frequency one. This is a remarkable application of the odd-frequency pairing concept when one can reinterpret the well-known resonance structure.

In the present study, we clarified the details of the proximity effect of the odd-frequency pairing state induced at the $\mathrm{N} / \mathrm{S}$ boundary. We reinterpreted the appearance of the MARS in terms of the enhanced odd-frequency pair amplitude. Though we explicitly studied N/S junctions only, the oddfrequency pairing state is also expected near impurities and within Abrikosov vortex cores, where the amplitude of the pair potential is reduced. The present result indicates the ubiquitous presence of odd-frequency pairing states because most real superconductors are not uniform. That means that the odd-frequency pairing is not at all a rare situation as was previously assumed. Thus we believe that the odd-frequency pairing may become an important concept in understanding the physics of nonuniform superconducting systems.

In the present paper, the proximity effect is studied in the ballistic limit. In the present case, the enhanced oddfrequency pair amplitude appears in the $\mathrm{N}$ region for both $p_{x}$-wave and $d_{x y}$-wave junctions. It is very interesting to study in the intermediate regime ${ }^{28}$ since the parities of these states are different. In the diffusive limit, the proximity effect 
survives only for the former case. There are several interesting phase-coherent effects relevant to MARS. ${ }^{29,30}$ These preexisting phenomena can be reinterpreted in terms of the oddfrequency pairing state.

\section{ACKNOWLEDGMENTS}

One of the authors (Y.T.) expresses his sincerest gratitude for discussions with M. Ueda, S. Kashiwaya, and K. Nagai.
This work is supported by a Grant-in-Aid for Scientific Research (Grants No. 17071007 and No. 17340106) from the Ministry of Education, Culture, Sports, Science and Technology of Japan, by the Japan Society for the Promotion of Science (JSPS), and by NanoNed Project No. TCS.7029. The computation in this work was done using the facilities of the Supercomputer Center, Institute for Solid State Physics, University of Tokyo. This work was supported by the NTT Basic Research Laboratory.
${ }^{1}$ V. L. Berezinskii, JETP Lett. 20, 287 (1974).

${ }^{2}$ A. Balatsky and E. Abrahams, Phys. Rev. B 45, 13125 (1992); E. Abrahams, A. Balatsky, D. J. Scalapino, and J. R. Schrieffer, ibid. 52, 1271 (1995).

${ }^{3}$ M. Vojta and E. Dagotto, Phys. Rev. B 59, R713 (1999).

${ }^{4}$ P. Coleman, E. Miranda, and A. Tsvelik, Phys. Rev. B 49, 8955 (1994); O. Zachar, S. A. Kivelson, and V. J. Emery, Phys. Rev. Lett. 77, 1342 (1996); P. Coleman, A. Georges, and A. M. Tsvelik, J. Phys.: Condens. Matter 9, 345 (1997).

${ }^{5}$ Y. Fuseya, H. Kohno, and K. Miyake, J. Phys. Soc. Jpn. 72, 2914 (2003).

${ }^{6}$ D. Belitz and T. R. Kirkpatrick, Phys. Rev. B 58, 8214 (1998); 46, 8393 (1992); 60, 3485 (1999); T. R. Kirkpatrick and D. Belitz, Phys. Rev. Lett. 66, 1533 (1991).

${ }^{7}$ F. S. Bergeret, A. F. Volkov, and K. B. Efetov, Phys. Rev. Lett. 86, 4096 (2001).

${ }^{8}$ F. S. Bergeret, A. F. Volkov, and K. B. Efetov, Rev. Mod. Phys. 77, 1321 (2005); A. Kadigrobov, R. I. Shekhter, and M. Jonson, Europhys. Lett. 90, 394 (2001); M. L. Kulic and M. Endres, Phys. Rev. B 62, 11846 (2000); Y. V. Fominov, A. A. Golubov, and M. Y. Kupriyanov, JETP Lett. 77, 510 (2003).

${ }^{9}$ M. Eschrig, J. Kopu, J. C. Cuevas, and G. Schön, Phys. Rev. Lett. 90, 137003 (2003).

${ }^{10}$ R. S. Keizer, S. T. B. Goennenwein, T. M. Klapwijk, G. Miao, G. Xiao, and A. Gupta, Nature (London) 439, 825 (2006); I. Sosnin, H. Cho, V. T. Petrashov, and A. F. Volkov, Phys. Rev. Lett. 96, 157002 (2006); V. Braude and Yu. V. Nazarov, ibid. 98, 077003 (2007); Y. Asano, Y. Tanaka and A. A. Golubov, ibid. 98, 107002 (2007).

${ }^{11}$ T. Yokoyama, Y. Tanaka, and A. A. Golubov, Phys. Rev. B 75, 134510 (2007).

${ }^{12}$ Y. Tanaka and A. A. Golubov, Phys. Rev. Lett. 98, 037003 (2007).

${ }^{13}$ Y. Tanaka and S. Kashiwaya, Phys. Rev. B 70, 012507 (2004); Y. Tanaka, S. Kashiwaya, and T. Yokoyama, ibid. 71, 094513 (2005); Y. Asano, Y. Tanaka, and S. Kashiwaya, Phys. Rev. Lett. 96, 097007 (2006); Y. Asano, Y. Tanaka, and A. A. Golubov, Phys. Rev. Lett. 99, 067005 (2007).

${ }^{14}$ Y. Tanaka and S. Kashiwaya, Phys. Rev. Lett. 74, 3451 (1995); S. Kashiwaya and Y. Tanaka, Rep. Prog. Phys. 63, 1641 (2000), T. Löfwander, V. S. Shumeiko, and G. Wendin, Semicond. Sci. Technol. 14, R53 (2001); Y. Asano, Y. Tanaka, and S. Kashiwaya, Phys. Rev. B 69, 134501 (2004).

${ }^{15}$ L. J. Buchholtz and G. Zwicknagl, Phys. Rev. B 23, 5788 (1981); J. Hara and K. Nagai, Prog. Theor. Phys. 74, 1237 (1986); C. R. Hu, Phys. Rev. Lett. 72, 1526 (1994); C. Bruder, Phys. Rev. B 41, 4017 (1990).

${ }^{16}$ L. Alff, H. Takashima, S. Kashiwaya, N. Terada, H. Ihara, Y.
Tanaka, M. Koyanagi, and K. Kajimura, Phys. Rev. B 55, R14757 (1997); M. Covington, M. Aprili, E. Paraoanu, L. H. Greene, F. Xu, J. Zhu, and C. A. Mirkin, Phys. Rev. Lett. 79, 277 (1997); J. Y. T. Wei, N.-C. Yeh, D. F. Garrigus, and M. Strasik, ibid. 81, 2542 (1998); I. Iguchi, W. Wang, M. Yamazaki, Y. Tanaka, and S. Kashiwaya, Phys. Rev. B 62, R6131 (2000); F. Laube, G. Goll, H. v. Lohneysen, M. Fogelström, and F. Lichtenberg, Phys. Rev. Lett. 84, 1595 (2000); Z. Q. Mao, K. D. Nelson, R. Jin, Y. Liu, and Y. Maeno, ibid. 87, 037003 (2001)

${ }^{17}$ Y. Tanaka, A. A. Golubov, S. Kashiwaya, and M. Ueda, Phys. Rev. Lett. 99, 037005 (2007).

${ }^{18}$ M. Eschrig, T. Lofwander, Th. Champel, J. C. Cuevas, and G. Schon, J. Low Temp. Phys. 147, 457 (2007).

${ }^{19}$ Y. Nagato and K. Nagai, Phys. Rev. B 51, 16254 (1995); L. J. Buchholtz, M. Palumbo, D. Rainer, and J. A. Sauls, J. Low Temp. Phys. 101, 1097 (1995); Yu. S. Barash, A. A. Svidzinsky, and H. Burkhardt, Phys. Rev. B 55, 15282 (1997).

${ }^{20}$ M. Eschrig, Phys. Rev. B 61, 9061 (2000); A. Shelankov and M. Ozana, ibid. 61, 7077 (2000); N. Schopohl and K. Maki, ibid. 52, 490 (1995); C. Iniotakis, S. Graser, T. Dahm, and N. Schopohl, ibid. 71, 214508 (2005).

${ }^{21}$ K. Nagai (unpublished); M. Ashida, S. Aoyama, J. Hara, and K. Nagai, Phys. Rev. B 40, 8673 (1989); Y. Nagato, K. Nagai, and J. Hara, J. Low Temp. Phys. 93, 33 (1993); J. Kurkijärvi and D. Rainer, in Helium Three, edited by W. P. Halperin and L. P. Pitaevskii (Elsevier, Amsterdam, 1990); Y. Tanuma, Y. Tanaka, and S. Kashiwaya, Phys. Rev. B 64, 214519 (2001).

${ }^{22}$ J. W. Serene and D. Rainer, Phys. Rep. 101, 221 (1983).

${ }^{23}$ G. Eilenberger, Z. Phys. 214, 195 (1968).

${ }^{24}$ M. Matsumoto and H. Shiba, J. Phys. Soc. Jpn. 64, 3384 (1995).

${ }^{25}$ W. L. McMillan, Phys. Rev. 175, 537 (1968).

${ }^{26}$ J. M. Rowell and W. L. MaMillan, Phys. Rev. Lett. 16, 453 (1966); J. M. Rowell, ibid. 30, 167 (1973).

${ }^{27}$ Y. Tanaka, Y. V. Nazarov, and S. Kashiwaya, Phys. Rev. Lett. 90, 167003 (2003); Y. Tanaka, Y. V. Nazarov, A. A. Golubov, and S. Kashiwaya, Phys. Rev. B 69, 144519 (2004).

${ }^{28}$ T. Löfwander, Phys. Rev. B 70, 094518 (2004).

${ }^{29}$ Y. Tanaka and S. Kashiwaya, Phys. Rev. B 53, R11957 (1996); 56, 892 (1997); Yu. S. Barash, H. Burkhardt, and D. Rainer, Phys. Rev. Lett. 77, 4070 (1996).

${ }^{30}$ S. Higashitani, J. Phys. Soc. Jpn. 66, 2556 (1997); Yu. S. Barash, M. S. Kalenkov, and J. Kurkijärvi, Phys. Rev. B 62, 6665 (2000); H. Walter, W. Prusseit, R. Semerad, H. Kinder, W. Assmann, H. Huber, H. Burkhardt, D. Rainer, and J. A. Sauls, Phys. Rev. Lett. 80, 3598 (1998); Y. Tanaka, Y. Asano, A. A. Golubov, and S. Kashiwaya, Phys. Rev. B 72, 140503(R) (2005). 